\title{
Splicing variant of WDFY4 augments MDA5 signaling and the risk of clinically amyopathic dermatomyositis
}

DOI:

10.1136/annrheumdis-2017-212149

\section{Document Version}

Accepted author manuscript

Link to publication record in Manchester Research Explorer

\section{Citation for published version (APA):}

Chinoy, H., Rothwell, S., Lamb, J., \& et al (2018). Splicing variant of WDFY4 augments MDA5 signaling and the risk of clinically amyopathic dermatomyositis. Annals of the rheumatic diseases.

https://doi.org/10.1136/annrheumdis-2017-212149

\section{Published in:}

Annals of the rheumatic diseases

\section{Citing this paper}

Please note that where the full-text provided on Manchester Research Explorer is the Author Accepted Manuscript or Proof version this may differ from the final Published version. If citing, it is advised that you check and use the publisher's definitive version.

\section{General rights}

Copyright and moral rights for the publications made accessible in the Research Explorer are retained by the authors and/or other copyright owners and it is a condition of accessing publications that users recognise and abide by the legal requirements associated with these rights.

\section{Takedown policy}

If you believe that this document breaches copyright please refer to the University of Manchester's Takedown Procedures [http://man.ac.uk/04Y6Bo] or contact uml.scholarlycommunications@manchester.ac.uk providing relevant details, so we can investigate your claim.

\section{OPEN ACCESS}




\section{sansoster \\ RHEUMATIC DISEASES \\ The Eular Journal}

\section{Splicing variant of WDFY4 augments MDA5 signaling and the risk of clinically amyopathic dermatomyositis}

\begin{tabular}{|c|c|}
\hline Journal: & Annals of the Rheumatic Diseases \\
\hline Manuscript ID & annrheumdis-2017-212149.R2 \\
\hline Article Type: & Extended report \\
\hline Date Submitted by the Author: & 30-Dec-2017 \\
\hline Complete List of Authors: & $\begin{array}{l}\text { Kochi, Yuta; Rikagaku Kenkyujo Togo Seimei Ikagaku Kenkyu Center, } \\
\text { Laboratory for Autoimmune Diseases } \\
\text { Kamatani, Yoichiro; RIKEN Center for Integrative Medical Sciences } \\
\text { Kondo, Yuya; University of Tsukuba, Department of Internal Medicine, } \\
\text { Faculty of Medicine } \\
\text { Suzuki, Akari; Rikagaku Kenkyujo Togo Seimei Ikagaku Kenkyu Center, } \\
\text { Laboratory for Autoimmune Diseases } \\
\text { Kawakami, Eiryo; RIKEN Center for Integrative Medical Sciences } \\
\text { Hiwa, Ryosuke; Kyoto University Graduate School of Medicine } \\
\text { Momozawa, Yukihide; Rikagaku Kenkyujo Yokohama Campus } \\
\text { Fujimoto, Manabu; University of Tsukuba, Department of Dermatology } \\
\text { Jinnin, Masatoshi } \\
\text { Tanaka, Yoshiya; University of Occupational and Environmental Health, } \\
\text { Japan, First Department of Internal Medicine } \\
\text { Kanda, Takashi; Yamaguchi University, Neurology } \\
\text { Cooper, Robert; University of Liverpool } \\
\text { Chinoy, Hector; The University of Manchester, Centre for Musculoskeletal } \\
\text { Research } \\
\text { Rothwell, Simon; University of Manchester, Centre for Genetics and } \\
\text { Genomics, Arthritis Research UK } \\
\text { Lamb, Janine; The University of Manchester, Centre for Integrated } \\
\text { Genomic Medical Research, Manchester Academic Health Science Centre } \\
\text { Vencovský, Jiři; Charles University } \\
\text { Mann, Herman; Institute of Rheumatology, } \\
\text { Ohmura, Koichiro; Kyoto University, Rheumatology and Clinical } \\
\text { Immunology } \\
\text { Myouzen, Keiko; Rikagaku Kenkyujo Yokohama Campus } \\
\text { Ishigaki, Kazuyoshi; the University of Tokyo, Allergy and Rheumatology } \\
\text { Nakashima, Ran; Kyoto University, Rheumatology and Clinical Immunology } \\
\text { Hosono, Yuji; Kyoto University, Rheumatology and Clinical Immunology } \\
\text { Tsuboi, Hiroto; University of Tsukuba, Division of Clinical Immunology, } \\
\text { Doctoral Program in Clinical Sciences, Graduate School of Comprehensive } \\
\text { Human Sciences } \\
\text { Kawasumi, Hidenaga; Tokyo Joshi Ika Daigaku } \\
\text { Iwasaki, Yukiko; Graduate School of Medicine, the University of Tokyo, } \\
\text { Department of Allergy and Rheumatology } \\
\text { Kajiyama, Hiroshi; Saitama Ika Daigaku } \\
\text { Horita, Tetsuya; Hokkaido University, Graduate School of Medicine }\end{array}$ \\
\hline
\end{tabular}




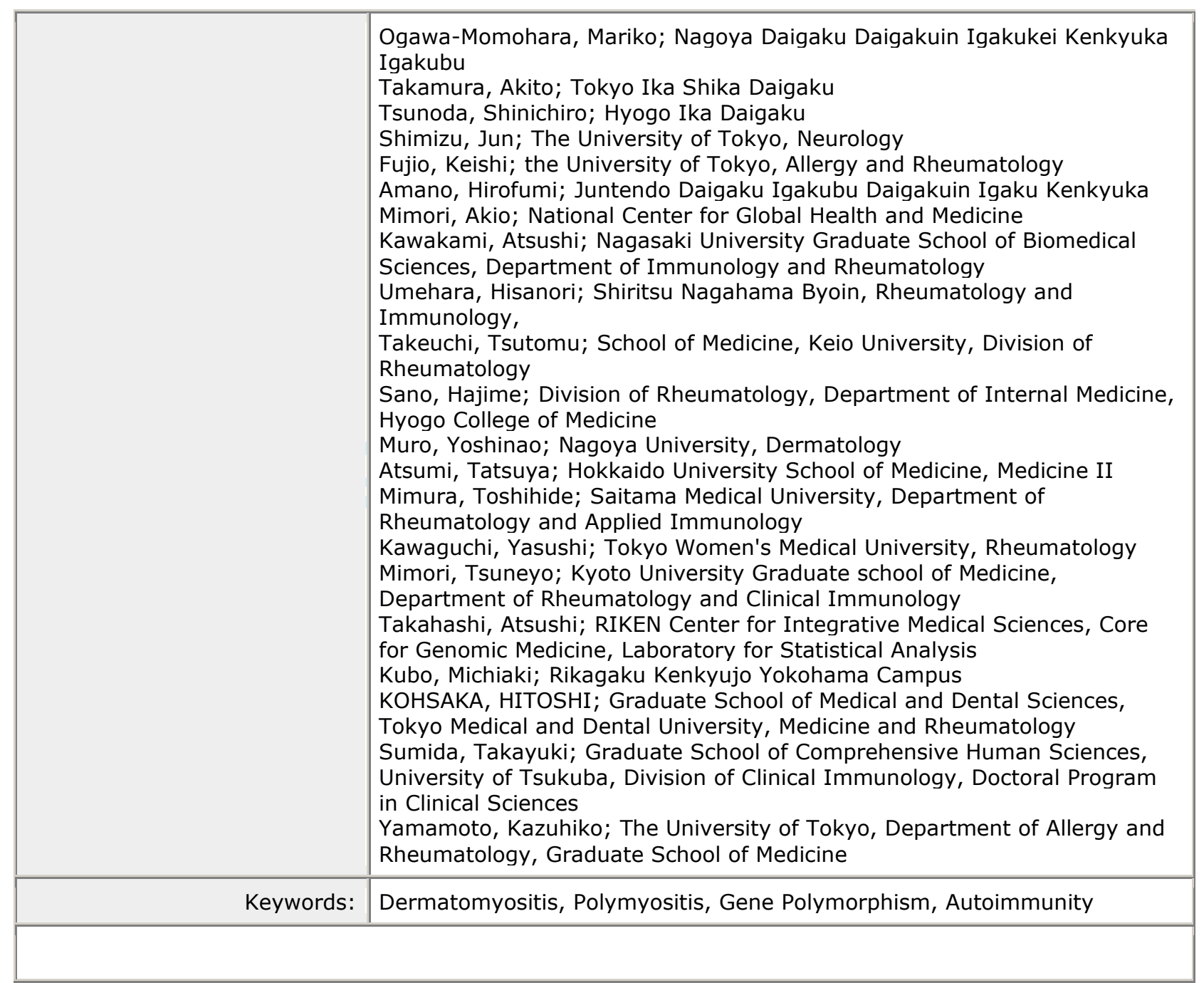

\section{SCHOLARONE ${ }^{m}$}

Manuscripts 


\section{(Splicing variant of WDFY4 augments MDA5 signaling and the risk of clinically amyopathic dermatomyositis}

\section{Authors}

Yuta Kochi ${ }^{1}$, Yoichiro Kamatani ${ }^{2}$, Yuya Kondo ${ }^{3}$, Akari Suzuki ${ }^{1}$, Eiryo Kawakami ${ }^{4}$, Ryosuke Hiwa, Yukihide Momozawa ${ }^{6}$, Manabu Fujimoto, ${ }^{7,8}$ Masatoshi Jinnin ${ }^{9}$, Yoshiya Tanaka ${ }^{10}$, Takashi Kanda ${ }^{11}$, Robert G. Cooper ${ }^{12,13}$, Hector Chinoy ${ }^{14,15}$, Simon Rothwell ${ }^{15}$, Janine A. Lamb ${ }^{13}$, Jiří Vencovský ${ }^{16}$, Heřman Mann ${ }^{16}$, Koichiro Ohmura ${ }^{5}$, Keiko Myouzen ${ }^{1}$, Kazuyoshi Ishigaki ${ }^{2}$, Ran Nakashima ${ }^{5}$, Yuji Hosono ${ }^{5}$, Hiroto Tsuboi ${ }^{3}$, Hidenaga Kawasumi ${ }^{17}$, Yukiko Iwasaki ${ }^{18}$, Hiroshi Kajiyama ${ }^{19}$, Tetsuya Horita ${ }^{20}$, Mariko Ogawa-Momohara $^{21}$, Akito Takamura ${ }^{22}$, Shinichiro Tsunoda ${ }^{23}$, Jun Shimizu ${ }^{24}$, Keishi Fujio $^{18}$, Hirofumi Amano ${ }^{25}$, Akio Mimori ${ }^{26}$, Atsushi Kawakami ${ }^{27}$, Hisanori Umehara ${ }^{28}$, Tsutomu Takeuchi ${ }^{29}$, Hajime Sano ${ }^{23}$, Yoshinao Muro ${ }^{21}$, Tatsuya Atsumi ${ }^{20}$, Toshihide Mimura $^{19}$, Yasushi Kawaguchi ${ }^{17}$, Tsuneyo Mimori ${ }^{5}$, Atsushi Takahashi ${ }^{2}$, Michiaki Kubo $^{6}$, Hitoshi Kohsaka ${ }^{22}$, Takayuki Sumida ${ }^{3}$, Kazuhiko Yamamoto ${ }^{1,18}$

\section{Corresponding to}

Yuta Kochi, MD, PhD.

Laboratory for Autoimmune Diseases, RIKEN Center for Integrative Medical Sciences 1-7-22 Suehiro-Cho, Tsurumi-Ku, Yokohama, 230-0045, Japan

yuta.kochi@riken.jp

\footnotetext{
Affiliations

${ }^{1}$ Laboratory for Autoimmune Diseases, RIKEN Center for Integrative Medical Sciences, Yokohama, Japan.

${ }^{2}$ Laboratory for Statistical Analysis, RIKEN Center for Integrative Medical Sciences, Yokohama, Japan.

${ }^{3}$ Department of Internal Medicine, Faculty of Medicine, University of Tsukuba, Tsukuba, Japan.

${ }^{4}$ Laboratory for Disease Systems Modeling, RIKEN Center for Integrated Medical Sciences, Yokohama, Japan.

${ }^{5}$ Department of Rheumatology and Clinical Immunology, Kyoto University Graduate School of Medicine, Kyoto, Japan.

${ }^{6}$ Laboratory for Genotyping Development, RIKEN Center for Integrative Medical Sciences, Yokohama, Japan.

${ }^{7}$ Department of Dermatology, Faculty of Medicine, Institute of Medical, Pharmaceutical and Health Sciences, Kanazawa University, Kanazawa, Japan.

${ }^{8}$ Department of Dermatology, University of Tsukuba, Ibaraki, Japan.

${ }^{9}$ Department of Dermatology and Plastic Surgery, Faculty of Life Sciences, Kumamoto University, Kumamoto, Japan.
} 
${ }^{10}$ The First Department of Internal Medicine, University of Occupational and Environmental Health, Kitakyushu, Japan.

${ }^{11}$ Department of Neurology and Clinical Neuroscience, Yamaguchi University Graduate School of Medicine, Ube, Japan.

${ }^{12}$ MRC-ARUK Institute for Ageing and Chronic Disease, University of Liverpool, Liverpool, UK.

${ }^{13}$ Centre for Integrated Genomic Medical Research, Division of Population Health, Health Services Research and Primary Care, School of Health Sciences, Faculty of Biology, Medicine and Health, Manchester Academic Health Science Centre, The University of Manchester, Manchester, UK.

${ }^{14}$ Rheumatology Department, Manchester Academic Health Science Centre, Salford Royal NHS Foundation Trust, Salford, UK.

${ }^{15}$ The National Institute for Health Research Manchester Musculoskeletal Biomedical Research Unit, Central Manchester University Hospitals NHS Foundation Trust, Manchester Academic Health Science Centre, The University of Manchester, Manchester, UK.

${ }^{16}$ Institute of Rheumatology, Charles University, Prague, Czech Republic.

${ }^{17}$ Institute of Rheumatology, Tokyo Women's Medical University, Tokyo, Japan.

${ }^{18}$ Department of Allergy and Rheumatology, Graduate School of Medicine, the University of Tokyo, Tokyo, Japan.

${ }^{19}$ Department of Rheumatology and Applied Immunology, Faculty of Medicine, Saitama Medical University, Saitama, Japan.

${ }^{20}$ Department of Rheumatology, Endocrinology and Nephrology, Faculty of Medicine and Graduate School of Medicine, Hokkaido University, Sapporo, Japan.

${ }^{21}$ Department of Dermatology, Nagoya University Graduate School of Medicine, Nagoya, Japan.

${ }^{22}$ Department of Rheumatology, Graduate School of Medical and Dental Sciences, Tokyo Medical and Dental University, Tokyo, Japan.

${ }^{23}$ Division of Rheumatology Department of Internal Medicine, Hyogo College of Medicine, Hyogo, Japan.

${ }^{24}$ Department of Neurology, Graduate School of Medicine, The University of Tokyo, Japan.

${ }^{25}$ Department of Internal Medicine and Rheumatology, Juntendo University School of Medicine, Tokyo, Japan.

${ }^{26}$ Division of Rheumatic Diseases, National Center for Global Health and Medicine, Tokyo, Japan.

${ }^{27}$ Department of Immunology and Rheumatology, Unit of Advanced Preventive Medical Sciences, Nagasaki University Graduate School of Biomedical Sciences, Nagasaki, Japan.

${ }^{28}$ Department of Hematology and Immunology, Kanazawa Medical University, Ishikawa, Japan

${ }^{29}$ Division of Rheumatology, Department of Internal Medicine, Keio University School of Medicine, Tokyo, Japan. 


\begin{abstract}
Objectives Idiopathic inflammatory myopathies (IIM) are a heterogeneous group of rare autoimmune diseases in which both genetic and environmental factors play important roles. To identify genetic factors of IIM including polymyositis (PM), dermatomyositis (DM), and clinically amyopathic DM (CADM), we performed the first genome-wide association study (GWAS) for IIM in an Asian population.

Methods We genotyped and tested 496,819 SNPs for association using 576 IIM patients and 6,270 control subjects. We also examined the causal mechanism of disease associated variants by in silico analyses using publicly available data sets as well as by in in vitro analyses using reporter assays and apoptosis assays.
\end{abstract}

Results We identified a variant in WDFY4 that was significantly associated with CADM (rs7919656; odds ratio $\left.(\mathrm{OR})=3.87 ; P=1.5 \times 10^{-8}\right)$. This variant had a cis-splicing quantitative trait locus (QTL) effect for a truncated WDFY4 isoform (tr-WDFY4), with higher expression in the risk allele. Trans-expression QTL (eQTL) analysis of this variant showed a positive correlation with the expression of NF- $\kappa \mathrm{B}$ associated genes. Furthermore, we demonstrated that both WDFY4 and tr-WDFY4 interacted with pattern recognition receptors such as TLR3, TLR4, TLR9, and MDA5 and augmented the NF- $\kappa B$ activation by these receptors. WDFY4 isoforms also enhanced MDA5-induced apoptosis, to a greater extent in the tr-WDFY4-transfected cells.

Conclusions As CADM is characterized by the appearance of anti-MDA5 autoantibodies and severe lung inflammation, the WDFY4 variant may play a critical role in the pathogenesis of CADM. 


\section{INTRODUCTION}

Idiopathic inflammatory myopathies (IIM) are autoimmune conditions characterized by muscle weakness and inflammation, and the most common types are polymyositis (PM) and dermatomyositis (DM). ${ }^{12}$ Clinically amyopathic DM (CADM), a subset of DM, lacks distinct muscle features but often manifests rapidly progressive interstitial pneumonia (RPIP). ${ }^{3-6}$ The mortality rate of CADM at 6 months from diagnosis reaches approximately $50 \%$, making it one of the most fatal autoimmune diseases. ${ }^{78}$ The appearance of autoantibodies such as anti-MDA5-antibodies in CADM suggests a role for autoimmunity in the pathogenesis. ${ }^{9-11}$ Although the etiology of IIM remains unknown, virus infection may be the major environmental factor. ${ }^{12}$ In addition, IIM patients and their close relatives are more likely to develop other autoimmune diseases, suggesting shared genetic factors. ${ }^{13-15}$ In fact, a previous genome-wide association studies (GWAS) and candidate gene analyses in European populations demonstrated multiple loci that were shared with other autoimmune diseases. ${ }^{15-18}$

However, when compared with other autoimmune diseases, little is known about the genetic background of IIM. This may be due to the rarity of disease, precluding researchers from designing sufficiently powered studies. In addition, because IIMs are a heterogenous group of rare disorders, an overall pooled analysis might also reduce the power for detecting loci specific to certain subtypes. Therefore, as suggested ${ }^{19}$, studies of disease subsets are needed to increase the power for detecting subset-specific genetic components. Here, to elucidate genetic loci associated with IIM, we performed a GWAS for IIM in the Japanese population. This study was the first GWAS for IIM in an Asian population. We also performed a GWAS for CADM as a subset analysis, which was the first such study in the world. 


\section{Methods}

\section{Subjects}

We enrolled 592 IIM cases (mean age, 55.5 \pm 14.2 years; female, 74.6\%) from 18 medical institutes in Japan. PM/DM fulfilled the definite or probable diagnostic criteria of Bohan and Peter. ${ }^{1}$ The diagnosis of CADM was based on the criteria by Sontheimer et al. ${ }^{36}$ We enrolled 6270 control subjects without history of autoimmune diseases (mean age, $57.0 \pm 13.8$ years; female, $48.2 \%$ ) through the Biobank project ${ }^{20}$. The European subjects (21 CADM cases) were recruited as part of an international myositis genetics consortium (MYOGEN), ${ }^{17}$ and four controls for each case (84 controls) were matched for ethnicity using principal components analysis (PCA) coordinates. ${ }^{21}$ All subjects were provided with written informed consent for participation in the study as approved by the ethical committee of each institutional review board.

\section{GWAS}

We genotyped case samples using HumanOmniExpressExome BeadChip and control samples using HumanOmniExpress BeadChip and HumanExome BeadChip (Illumina). For quality control, we excluded single nucleotide polymorphisms (SNPs) with a call rate $<0.99$, a mean allele frequency $(\mathrm{MAF})<0.05$, and those not in Hardy-Weinberg equilibrium in controls $\left(P<1 \times 10^{-6}\right)$. We removed samples that showed second-degree relatedness or closer by an identity-by-state method. We performed PCA using four reference populations from HapMap data including CEU, YRI, JPT and CHB using SmartPCA software. ${ }^{22}$ We identified outliers not belonging to the JPT/CHB cluster by using the top two principal components. After this screening, we selected 576 cases and 6270 controls for subsequent analysis. This sample size had statistical power $>0.5$ for 
detecting common variants with moderate effects $(\mathrm{MAF}=0.5$ and $\mathrm{OR}=1.5)$ as calculated by QUANTO. ${ }^{23}$ The European samples were genotyped by the Immunochip (Illumina) ${ }^{18}$. We performed genotype imputation within the WDFY4 locus by Minimac ${ }^{24}$ using the 1000 Genomes Project Asian data as reference.

\section{eQTL analysis}

Both cis- and trans- eQTL analyses were performed using Matrix eQTL with the linear model. ${ }^{25}$ We used the Geuvadis project data ${ }^{26}$ of European samples $(n=373)$, “GD462.GeneQuantRPKM.50FN.samplename.resk10.norm.txt” for the gene level eQTL analysis and "GD462.ExonQuantCount.45N.50FN.samplename.resk10.txt" for the exon level eQTL analysis.

\section{WPGSA}

To predict the activities of transcriptional regulators responsible for observed gene expression changes resulting from trans-eQTL effects of tr-WDFY4, we used weighted parametric gene set analysis (wPGSA) ${ }^{27}$ with some modifications (supplementary figure S7). ChIP-seq data of multiple types of human cells were obtained from the GEO-database. In total, 3082 ChIP-seq data of 431 TFs were used. We used the trans-eQTL effects of WDFY4 variant (rs7919656) on every gene ( $\beta$ obtained by Matrix-eQTL) as input, instead of the log-fold change of the gene expression.

\section{Reporter assay}

The coding sequences of WDFY4 and tr-WDFY4 were cloned into the pcDNA3.1D/V5-His-TOPO vector using cDNAs from EBV-immortalized B cells. 
Human pattern recognition receptors were cloned into the pEF-BOS-FLAG vector (TLR3 was provided by Dr. Matsumoto, Hokkaido University; TLR4 and TLR9 were provided by Dr. Miyake, the University of Tokyo). UNC93B1 was cloned into the pcDNA5/FRT/V5-His vector. UNC93B1 was co-transfected for the experiments of TLR7 or TLR9, because it is essential to deliver these receptors to endolysosomes. ${ }^{28} \mathrm{We}$ cultured HEK-293 cells in DMEM supplemented with 10\% fetal bovine serum. We

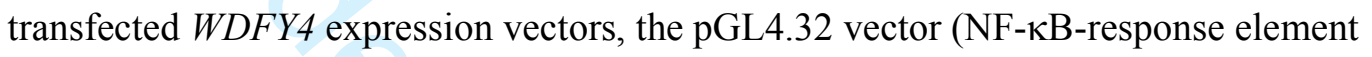
and luc2P) and the pGL4.74 vector (hRluc/TK) (Promega) into cells using the X-tremeGENE HP Transfection Reagent (Roche). The p55C1B-Luc vector ${ }^{29}$ (provided $^{2}$ by Dr. Fujita, Kyoto University) was used instead of pGL4.32 for IRF3 reporter assays. After 18h incubation, the cells were stimulated with poly (I:C) (Enzo Life Sciences), LPS (Sigma-Aldrich), imiquimod (InvivoGen), ODN2006 (InvivoGen), or high molecular weight poly (I:C) ${ }^{30}$ (provided by Dr. Fujita) for $4 \mathrm{~h}$. We then collected the cells and measured luciferase activity using Dual-Luciferase Reporter Assays according to the manufacturer's protocol (Promega).

\section{Immunoprecipitation and western blotting}

HEK-293 cells were transfected with each WDFY4 isoform and PRR and cultured for 24h. The cells were lysed in RIPA buffer ${ }^{31}$ and then incubated with Dynabeads protein-G (Life Technologies) coated with anti-FLAG-antibody (Sigma-Aldrich) and incubated at $4^{\circ} \mathrm{C}$ for $30 \mathrm{~min}$. The beads were then washed and boiled in SDS buffer before electrophoresis through NuPAGE Tris-Acetate gels. The proteins were transferred to PDVF membranes (Millipore), which were then probed with anti-V5-antibody (Invitrogen), visualized using ECL reagent (GE Healthcare), and 
detected using LAS-3000 (Fuji Film). We also performed immunoprecipitation using the sera from patients, instead of anti-FLAG-antibody.

\section{Apoptosis assay}

HepG2 Cells were transfected with each WDFY4 isoform using the Lipofectamine 3000 reagent (Invitrogen). The same vector with cloned $L a c Z$ was also transfected as a mock. Cells were incubated for $24 \mathrm{~h}$ and stimulated with $5 \mu \mathrm{g}$ of poly (I:C). The cells were permeabilized and stained with CaspACE FITC-VAD-FMK (Promega) and anti-His-Alexa 647. Caspase activity was evaluated in the His-positive cells using the BD Accuri C6 flow cytometer (BD Biosciences) to detect apoptotic cells.

\section{Immunofluorescence staining}

HEK-293 cells were grown on coverslips and transfected with the vectors for WDFY4 isoforms and MDA5. Cells were fixed in 4\% paraformaldehyde, permeabilized in $0.25 \%$ Triton X-100, and stained with anti-V5 and anti-mouse IgG-Alexa488 (Invitrogen) for WDFY4 and anti-FLAG-Cy3 (Sigma-Aldrich) for MDA5 detection. DAPI was used for counter-staining. Images were acquired with the confocal microscope N-SIM (Nikon) using $100 \mathrm{X}$ objective lens. The data were analyzed in 3D-SIM mode by using NIS-Elements software (Nikon).

\section{Measurement of anti-MDA5}

We measured anti-MDA5 autoantibodies in the sera from the CADM patients (18 sera were available) by protein-immunoprecipitation. ${ }^{9}$ 


\section{Statistics}

We tested the association of SNPs by allelic test using Plink $1.9,{ }^{32}$ setting the genome-wide significance threshold at $\alpha=5 \times 10^{-8}$. We used a quantile-quantile plot to evaluate the potential effects of population stratification. We used analysis of variance (ANOVA) with post-hoc Tukey's test in the reporter assays and apoptosis assays. 


\section{RESULTS \\ GWAS for IIM}

We genotyped the Japanese samples using Illumina SNP arrays for over 960,000 SNPs. After quality control filtering, the effective sample size was 576 IIM cases and 6,270 controls, and 496,819 SNPs were tested. The genomic inflation factor $\lambda_{G C}$ was 1.024 (supplementary figure S1), suggesting a negligible effect of population stratification. Cases comprised $236 \mathrm{PM}$ and $340 \mathrm{DM}$ patients including $33 \mathrm{CADM}$ patients, and GWASs for these disease subsets were also performed. No significant association signal $\left(P<5 \times 10^{-8}\right)$ was found in any of total IIM, PM or DM (supplementary figure S2). However, when CADM patients were evaluated alone, a significant association was observed for an intronic SNP of WDFY4 (WDFY Family Member 4; rs7919656; $\left.\mathrm{OR}=3.87 ; 95 \% \mathrm{CI}=2.23-6.55 ; P=1.5 \times 10^{-8}\right)$ (figure $1 \mathrm{~A}$ and table 1$)$. This SNP showed a nominal association with IIM, even when CADM patients were excluded $(P=0.037)$. In European CADM patients, although no obvious association signal was observed in rs7919656, distinct association signals $\left(r^{2}<0.2\right.$ with $r$ 7919656) were observed for the neighboring variants (rs11101462, $P=0.0092$; rs2889697, $P=0.0058$, supplementary figure S3)

\section{Splicing QTL effect of WDFY4 variant on truncated isoform}

To unravel the causal mechanism of the WDFY4 locus in this disease subset, we first searched for candidate functional variants. Among the variants in strong linkage disequilibrium (LD) with rs7919656 $\left(r^{2}>0.8\right)$ in the 1000 genome project Asian samples, there is no coding variant that altered the amino-acid sequences of neighboring genes (supplementary figure S4). We next searched the expression quantitative trait locus 
(eQTL) data of lymphoblastoid B-cells provided by the Geuvadis project. ${ }^{26}$ When correlations of the SNP (rs7919656) genotype and expression of both the gene and exons were evaluated, a significant eQTL effect for an alternative exon of WDFY4 was found (figure 1B). As no obvious eQTL effect was observed for expression of the whole gene or of neighboring exons (supplementary figure S5) this variant or variants in LD may have a splicing QTL effect for this WDFY4 isoform. Among such variants, two SNPs in intron 35 (rs10776647/rs10776648; figure 1C and supplementary figure S4) were candidate causal variants in this region; however, they did not disrupt classical splicing associated motifs. The alternative exon extends exon 36 of WDFY4, which introduces an alternate stop codon and truncates the protein (figure $1 \mathrm{C}, \mathrm{D})$ ). We verified the existence of this truncated isoform (tr-WDFY4) by cloning it with cDNA from EBV-immortalized B cells and expressing it in HEK-293 cells (figure 1E).

\section{Trans eQTL effect of WDFY4 variant on NF-kB activity}

WDFY4 encodes WDFY family member 4, which has a predicted BEACH domain and five WD domains at the C-terminal $\operatorname{side}^{33}$ (figure 1D). Although WDFY4 is expressed in dendritic cells, neutrophils, B-cells and macrophages (supplementary figure S6), to our knowledge, there have been no studies into its protein function. To obtain insight regarding the function of $W D F Y 4$, we performed trans-eQTL analysis of the variant using the same expression data of lymphoblastoid B-cells. A total of 387 genes were correlated with the number of risk alleles (uncorrected $P<0.05$ ), although no gene displayed genome-wide significant correlation (FDR $<0.05$ ). Notably, 279 genes (72.1\%) were positively correlated, indicating that upregulated expression of tr-WDFY4 results in upregulation of these genes. Among the top 10 genes with the largest 
trans-eQTL effects ( $\beta$ as calculated in the Matrix eQTL analysis), TYK2 and PTPN6 are well known regulators of signal pathways in B-cells (figure 2A). We then sought to identify the transcriptional factors (TFs) present downstream of tr-WDFY4 by weighted parametric gene set analysis (wPGSA). ${ }^{27}$ wPGSA utilizes all available ChIP-seq data obtained from multiple types of human cells and predicts the activities of transcriptional regulators responsible for observed gene expression changes (figure $2 \mathrm{~B}$ and supplementary figure S7). When trans-eQTL effects $(\beta)$ were used as input, among the $431 \mathrm{TFs}$ evaluated, $6 \mathrm{TFs}$ displayed significant associations ( $\mathrm{FDR}<0.05$ ) (figure $2 \mathrm{C}$ and supplementary table S1). We focused on TF65 (RELA/p65), because it comprises nuclear factor-kappa B (NF- $\kappa \mathrm{B})$ heterodimers that are one of the most important TFs in the immune system. ${ }^{34}$ Based on these findings, we assumed that the NF- $\kappa \mathrm{B}$ pathway is activated downstream of WDFY4. Indeed, NF- $\mathrm{BB}$ reporter assays showed that both WDFY4 and tr-WDFY4 enhanced NF-אB activity when transfected into HEK-293 cells with stimulation of TNF- $\alpha$ (figure 2D).

\section{WDFY4 augments signals of innate pattern recognition receptors}

These observations, as well as a recent report that WDFY1, another member of the WDFY gene family, augmented TLR3 and TLR9 signaling $^{35}$, motivated us to investigate the role of WDFY4 in PRR signaling. We chose TLR3/TLR4/TLR7/TLR9 and MDA5 for analysis considering their implied roles in disease pathogenesis ${ }^{43637}$, as well as their potential to activate NF- $\mathrm{NB} .{ }^{38} \mathrm{We}$ first performed $\mathrm{NF}-\kappa \mathrm{B}$ reporter assays by simultaneously transfecting each WDFY4 isoform with each PRR. While both WDFY4 isoforms enhanced NF- $\kappa B$ signaling of TLR3/TLR4/TLR9 and MDA5, they had a negligible effect on TLR7 signaling (figure 3A). Of note, tr-WDFY4 markedly 
enhanced the MDA5 signal when stimulated with poly (I:C), a synthetic analog of double-stranded RNA. We also examined the impact of WDFY4 isoforms on cytokine production by quantifying the levels of IL- 8 in the cell culture supernatants. Although both isoforms significantly increased the IL-8 production in steady state, the difference to mock transfectant became insignificant when cells were stimulated with poly (I:C) (supplementary figure S8). Next, we examined the physical interaction of WDFY4 isoforms and these PRRs by co-immunoprecipitation. Both V5-tagged WDFY4 isoforms interacted with these FLAG-tagged PRRs to greater or lesser degrees (figure 3B). The strongest interaction was observed between tr-WDFY4 and MDA5, which was consistent with the findings of reporter assays.

MDA5-mediated sensing of virus triggers not only endogenous immune responses but also cellular apoptosis to eliminate virus-infected cells. ${ }^{39}$ Therefore, to further evaluate the function of WDFY4 in the context of MDA5 signaling, we performed apoptosis assays. We used HepG2 cells for these assays, because stimulation of these cells with poly (I:C) is accompanied by upregulation of MDA5 and results in the induction of apoptosis $^{40}$ (figure 4A). When HepG2 cells were transfected with the WDFY4 isoforms and stimulated with/without poly (I:C), apoptosis was augmented to a greater extent in the tr-WDFY4-transfected cells than in the WDFY4-transfected cells (figure 4B). Again, this result is consistent with the observations regarding these isoforms in the NF- $\kappa B$ assays. As the apoptosis induced by MDA5 was reported to be independent of type I interferon signals ${ }^{41}$, we evaluated the effect of WDFY4 isoforms on type I interferon induction by co-transfected MDA5 using IRF3 reporter assays ${ }^{29}$. For comparison, we also examined TLR3 signaling. While no effect of WDFY4 isoforms on TLR3 induction of interferon was detected, MDA5 induction of interferon was markedly 
repressed when the WDFY4 isoforms were co-transfected with MDA5 (figure 4C).

These findings suggested that the WDFY4 isoforms are able to interact with MDA5 and alter its signaling in cells. This possibility was further supported by the observations that transfected WDFY4 isoforms and MDA5 co-localized in the cytoplasm of cells as assessed using immunofluorescence (figure 5A), and that the anti-MDA5-positive sera from patients could co-immunoprecipitate tr-WDFY4 proteins (figure 5B). 


\section{DISCUSSION}

We here demonstrated a significant association between a variant in WDFY4 and CADM susceptibility. Association of WDFY4 locus has been previously reported with systemic lupus erythematosus (SLE) ${ }^{42}$ and rheumatoid arthritis. ${ }^{43}$ A recent study reported that the SLE risk variant (rs2663052) also had a splicing QTL effect on WDFY4. ${ }^{44}$ However, rs2663052 is not in tight LD with the CADM risk variant (rs7919656, $r^{2}=0.46$ in the Asian samples), and rs2663052 displayed a weaker association signal in CADM in the present study $(P=0.0012)$. A missense variant (rs7097397), which is in moderate LD with rs7919656 $\left(r^{2}=0.58\right.$ in the Asian samples), may be another candidate causal variant in $\mathrm{SLE}^{42}$, but the association in CADM was moderate $(P=0.0022)$.

The fact that the CADM risk variant was also associated with upregulated expression of tr-WDFY4 suggested that WDFY4 was a strong candidate gene in this locus.

Although the biological function of WDFY4 was unknown, our analysis by wPGSA utilizing the trans-eQTL effects of $W D F Y 4$ variant implied that $\mathrm{NF}-\kappa \mathrm{B}$ was involved in the downstream regulation of WDFY4. Indeed, as demonstrated by in vitro assays, tr-WDFY4 augmented the NF- $\mathrm{BB}$ signals induced by PRRs including MDA5. Our in vitro assays also demonstrated that the intact WDFY4 augmented MDA5-induced $\mathrm{NF}-\kappa \mathrm{B}$ signal and apoptosis, but to lesser extent than tr-WDFY4. The differences in function between the intact WDFY4 and tr-WDFY4 should be further examined, but the lack of predicted WDFY4 protein domains (BEACH and WD domains) may have a marked impact on tr-WDFY4 function. Moreover, the suppression of IRF3 activity observed in the reporter assays suggests the role of WDFY4 in interferon signaling pathway. Because WDFY4 was shown to be downregulated in SLE patients ${ }^{45}$, WDFY4 
isoforms may control the balance between interferon and NF- $\kappa \mathrm{B}$ pathways, which should be further examined.

The lack of an association for rs7919656 (sQTL variant) in the European patients might be due to a lack of statistical power, because it diminished to 0.32 if a moderate effect size $(\mathrm{OR}=1.5)$ was assumed. However, as rs11101462, which displayed a positive association signal in Europeans, had an eQTL effect for WDFY4 in peripheral blood samples with higher expression in the risk allele (supplementary table S2), WDFY4 may also have a role in CADM in Europeans. The genetic heterogeneity observed between these populations might be partially explained by environmental factors. Because the extent of MDA5 signal transduction depends on the characteristic of ligands (viruses) such as their length ${ }^{30}$, the effects of WDFY4 isoforms on the MDA5 signaling pathway may also depend on the ligands. Therefore, if viruses trigger the disease and the type of viruses is different between the populations, the genetic role of the two variants and their related WDFY4 isoforms may also differ. The higher incidence of RPIP reported in Asian populations ${ }^{46}$ may indeed suggest etiological heterogeneity in CADM. An autoimmune reaction to MDA5 may be a central event in CADM pathogenesis, because CADM is characterized by the appearance of anti-MDA5-Abs. This phenomenon was also observed in $72.2 \%(n=13 / 18)$ of the CADM patients evaluated, and these anti-MDA5-positive sera from patients could co-immunoprecipitate tr-WDFY4 proteins. Interestingly, the serum from an anti-MDA5-negative patient could co-immunoprecipitiate tr-WDFY4 proteins, which might suggest the presence of anti-WDFY4 antibody. An inflammatory condition following exposure to antigens derived from apoptotic cells is one of the main mechanisms that trigger autoimmune disorders as shown in the animal models. ${ }^{47}$ Therefore, our findings that tr-WDFY4 
enhanced both inflammatory signals and apoptosis induced by MDA5 suggest that tr-WDFY4 has a critical role in the autoimmune response to MDA5 and possibly to WDFY4.

Moreover, because tr-WDFY4 augmented the NF- $\mathrm{KB}$ signals induced by PRRs, tr-WDFY4 may be also involved in the pathogenesis of RPIP. However, the high mortality rate of RPIP might have precluded the CADM patients with RPIP from participating in the study. Therefore, our samples might have underestimated the role of WDFY4 variant in this disease subset, particularly in patients with RPIP. To determine whether the WDFY4 variant can be used as a predictive biomarker for RPIP, a prospective study of new-onset CADM patients (or anti-MDA5-positive patients) is needed. Meanwhile, by assessing the environmental exposure, viral infections in particular, the role of gene-environmental interactions can be examined. As RPIP in CADM is still refractory to intense immunosuppressive therapies, tr-WDFY4 would be a candidate target in the treatment of CADM. 


\section{Acknowledgments}

We thank K. Kobayashi and M. Abe for their technical assistance and Dr. R. Yamada and Dr. Y. Okada for their advice at RIKEN. We thank Dr. F. W. Miller at the US National Institutes of Health, who established the MYOGEN consortium, for arranging the collaboration. We thank Dr. K. Miyake at the University of Tokyo, Dr. H. Kato and Dr. T. Fujita at Kyoto University for providing technical advice and reagents.

\section{Funding}

This work was supported by the Health and Labour Sciences Research Grants for research on intractable diseases (The Research Team for Autoimmune Diseases) from the Ministry of Health, Labour and Welfare of Japan. This work was also supported by the BioBank Japan Project of the Ministry of Education, Culture, Sports, Sciences and Technology of the Japanese government.

\section{Contributors}

Y.Kochi designed the study and drafted the manuscript. T.S., H.K., and Y.K. directed the Research Team for Autoimmune Diseases and revised the manuscript. Y.Momozawa. and M.K. performed the genotyping for GWAS. Y.Kochi, Y.Kamatani, and A.Takahashi analyzed the GWAS data. Y.Kochi, E.K., and K.I. performed eQTL analysis and wPGSA. Y.Kochi and A.S. conducted the reporter assays and immunoprecipitation. K.M. performed apoptosis assays and immunofluorescence staining. R.H., K.O., S.T., and T.M. assayed anti-MDA5 Abs and performed immunoprecipitation analysis using the sera from CADM patients. The following authors contributed to the Research Team for Autoimmune Diseases and its sample collection: Y.Kondo, M.F., M.J., Y.T., T.K., 
R.N., Y.H., H.T., H.Kawasumi, Y.I., H.Kajiyama, T.H., M.O-M., A.Takamura, S.T., J.S., K.F., H.A., A.M., A.K., H.U.,T.T., Y.Muro, T.A., T.Mimura, Y.Kawaguchi, T.Mimori, M.K., H.Kohsaka, T.S., and K.Y. M.K. directed the analysis of the BioBank samples.

R.G.C., H.C., J.V., and H.M. collected the European samples. S.R. and J.A.L. analyzed European CADM data.

\section{Conflict of interest}

The authors have declared that no conflict of interest exists. 


\section{REFERENCES}

1 Bohan A, Peter JB. Polymyositis and dermatomyositis (first of two parts). $N$ Engl $J$ Med 1975;292:344-7.

2 Dalakas MC. Inflammatory muscle diseases. N Engl J Med 2015;372:1734-47.

3 Sontheimer RD. Would a new name hasten the acceptance of amyopathic dermatomyositis (dermatomyositis siné myositis) as a distinctive subset within the idiopathic inflammatory dermatomyopathies spectrum of clinical illness? J Am Acad Dermatol 2002;46:626-36.

4 Mimori T, Nakashima R, Hosono Y. Interstitial lung disease in myositis: clinical subsets, biomarkers, and treatment. Current rheumatology reports 2012;14:264-74.

5 Sontheimer RD. MDA5 autoantibody-another indicator of clinical diversity in dermatomyositis. Annals of translational medicine 2017;5:160.

6 Gerami P, Schope JM, McDonald L, et al. A systematic review of adult-onset clinically amyopathic dermatomyositis (dermatomyositis sine myositis): a missing link within the spectrum of the idiopathic inflammatory myopathies. J Am Acad Dermatol 2006;54:597-613.

7 Koga T, Fujikawa K, Horai Y, et al. The diagnostic utility of anti-melanoma differentiation-associated gene 5 antibody testing for predicting the prognosis of Japanese patients with DM. Rheumatology (Oxford) 2012;51:1278-84.

8 Mukae H, Ishimoto H, Sakamoto N, et al. Clinical differences between interstitial lung disease associated with clinically amyopathic dermatomyositis and classic dermatomyositis. Chest 2009;136:1341-7.

9 Nakashima R, Imura Y, Kobayashi S, et al. The RIG-I-like receptor IFIH1/MDA5 is a dermatomyositis-specific autoantigen identified by the anti-CADM-140 antibody. Rheumatology (Oxford) 2010;49:433-40.

10 Senecal JL, Raynauld JP, Troyanov Y. Editorial: A New Classification of Adult Autoimmune Myositis. Arthritis Rheumatol 2017;69:878-84.

11 Satoh M, Tanaka S, Ceribelli A, et al. A Comprehensive Overview on Myositis-Specific Antibodies: New and Old Biomarkers in Idiopathic Inflammatory Myopathy. Clin Rev Allergy Immunol 2017;52:1-19.

12 Gan L, Miller FW. State of the art: what we know about infectious agents and myositis. Curr Opin Rheumatol 2011;23:585-94.

13 Ginn LR, Lin JP, Plotz PH, et al. Familial autoimmunity in pedigrees of idiopathic inflammatory myopathy patients suggests common genetic risk factors for many autoimmune diseases. Arthritis Rheum 1998;41:400-5.

14 Niewold TB, Wu SC, Smith M, et al. Familial aggregation of autoimmune disease in 
juvenile dermatomyositis. Pediatrics 2011;127:e1239-46.

15 Miller FW, Cooper RG, Vencovsky J, et al. Genome-wide association study of dermatomyositis reveals genetic overlap with other autoimmune disorders. Arthritis Rheum 2013;65:3239-47. Jani M, Massey J, Wedderburn LR, et al. Genotyping of immune-related genetic variants identifies TYK2 as a novel associated locus for idiopathic inflammatory myopathies. Ann Rheum Dis 2014;73:1750-2.

17 Miller FW, Chen W, O'Hanlon TP, et al. Genome-wide association study identifies HLA 8.1 ancestral haplotype alleles as major genetic risk factors for myositis phenotypes. Genes Immun 2015;16:470-80.

18 Rothwell S, Cooper RG, Lundberg IE, et al. Dense genotyping of immune-related loci in idiopathic inflammatory myopathies confirms HLA alleles as the strongest genetic risk factor and suggests different genetic background for major clinical subgroups. Ann Rheum Dis 2016;75:1558-66.

19 Bhattacharjee S, Rajaraman P, Jacobs KB, et al. A subset-based approach improves power and interpretation for the combined analysis of genetic association studies of heterogeneous traits. Am J Hum Genet 2012;90:821-35.

20 Nagai A, Hirata M, Kamatani Y, et al. Overview of the BioBank Japan Project: Study design and profile. J Epidemiol 2017;27:S2-S8.

21 Gregersen PK, Kosoy R, Lee AT, et al. Risk for myasthenia gravis maps to a (151) Pro-->Ala change in TNIP1 and to human leukocyte antigen-B*08. Ann Neurol 2012;72:927-35.

22 Price AL, Patterson NJ, Plenge RM, et al. Principal components analysis corrects for stratification in genome-wide association studies. Nat Genet 2006;38:904-9.

23 Gauderman WJ. Sample size requirements for matched case-control studies of gene-environment interaction. Stat Med 2002;21:35-50.

24 Howie B, Fuchsberger C, Stephens M, et al. Fast and accurate genotype imputation in genome-wide association studies through pre-phasing. Nat Genet 2012;44:955-9.

25 Shabalin AA. Matrix eQTL: ultra fast eQTL analysis via large matrix operations. Bioinformatics 2012;28:1353-8.

26 Lappalainen T, Sammeth M, Friedlander MR, et al. Transcriptome and genome sequencing uncovers functional variation in humans. Nature 2013;501:506-11.

27 Kawakami E, Nakaoka S, Ohta T, et al. Weighted enrichment method for prediction of transcription regulators from transcriptome and global chromatin immunoprecipitation data. Nucleic Acids Res 2016;44:5010-21.

28 Kim YM, Brinkmann MM, Paquet ME, et al. UNC93B1 delivers nucleotide-sensing 
toll-like receptors to endolysosomes. Nature 2008;452:234-8.

29 Devaraj SG, Wang N, Chen Z, et al. Regulation of IRF-3-dependent innate immunity by the papain-like protease domain of the severe acute respiratory syndrome coronavirus. J Biol Chem 2007;282:32208-21.

Kato H, Takeuchi O, Mikamo-Satoh E, et al. Length-dependent recognition of double-stranded ribonucleic acids by retinoic acid-inducible gene-I and melanoma differentiation-associated gene 5. J Exp Med 2008;205:1601-10.

31 Kochi Y, Myouzen K, Yamada R, et al. FCRL3, an autoimmune susceptibility gene, has inhibitory potential on B-cell receptor-mediated signaling. $J$ Immunol 2009;183:5502-10.

32 Purcell S, Neale B, Todd-Brown K, et al. PLINK: a tool set for whole-genome association and population-based linkage analyses. Am J Hum Genet 2007;81:559-75.

33 Cullinane AR, Schaffer AA, Huizing M. The BEACH is hot: a LYST of emerging roles for BEACH-domain containing proteins in human disease. Traffic 2013;14:749-66.

34 Vallabhapurapu S, Karin M. Regulation and function of NF-kappaB transcription factors in the immune system. Annu Rev Immunol 2009;27:693-733.

$35 \mathrm{Hu}$ YH, Zhang Y, Jiang LQ, et al. WDFY1 mediates TLR3/4 signaling by recruiting TRIF. EMBO reports 2015;16:447-55.

36 Brunn A, Zornbach K, Hans VH, et al. Toll-like receptors promote inflammation in idiopathic inflammatory myopathies. J Neuropathol Exp Neurol 2012;71:855-67.

37 Haq SA, Tournadre A. Idiopathic inflammatory myopathies: from immunopathogenesis to new therapeutic targets. Int J Rheum Dis 2015;18:818-25.

38 Kawai T, Akira S. Toll-like receptor and RIG-I-like receptor signaling. Ann $N$ Y Acad Sci 2008;1143:1-20.

39 Barral PM, Sarkar D, Su ZZ, et al. Functions of the cytoplasmic RNA sensors RIG-I and MDA-5: key regulators of innate immunity. Pharmacol Ther 2009;124:219-34.

40 Peng S, Geng J, Sun R, et al. Polyinosinic-polycytidylic acid liposome induces human hepatoma cells apoptosis which correlates to the up-regulation of RIG-I like receptors. Cancer Sci 2009;100:529-36.

41 Besch R, Poeck H, Hohenauer T, et al. Proapoptotic signaling induced by RIG-I and MDA-5 results in type I interferon-independent apoptosis in human melanoma cells. $J$ Clin Invest 2009;119:2399-411.

42 Yang W, Shen N, Ye DQ, et al. Genome-wide association study in Asian populations identifies variants in ETS1 and WDFY4 associated with systemic lupus erythematosus. PLoS genetics 2010;6:e1000841.

43 Okada Y, Wu D, Trynka G, et al. Genetics of rheumatoid arthritis contributes to 
biology and drug discovery. Nature 2014;506:376-81.

44 Odhams CA, Cortini A, Chen L, et al. Mapping eQTLs with RNA-seq reveals novel susceptibility genes, non-coding RNAs and alternative-splicing events in systemic lupus erythematosus. Hum Mol Genet 2017;26:1003-17.

45 Kuijpers TW, Vendelbosch S, Berg M, et al. Killer immunoglobulin receptor genes in spondyloarthritis. Curr Opin Rheumatol 2016;28:368-75.

46 Parronchi P, Radice A, Palterer B, et al. MDA5-positive dermatomyositis: an uncommon entity in Europe with variable clinical presentations. Clin Mol Allergy 2015;13:22.

47 Marshak-Rothstein A, Rifkin IR. Immunologically active autoantigens: the role of toll-like receptors in the development of chronic inflammatory disease. Annu Rev Immunol 2007;25:419-41. 
Table 1 Association analysis of rs7919656 with IIM

10

11

\begin{tabular}{|c|c|c|c|c|c|c|c|}
\hline \multirow{2}{*}{ Disease } & \multicolumn{2}{|c|}{ Numbers } & \multicolumn{2}{|c|}{ Allele A frequency } & \multicolumn{3}{|c|}{ Allelic comparison } \\
\hline & atients & Controls & Patients & Controls & OR ( 95\% Cl ) & $\chi^{2}$ & $P$ value \\
\hline IIM total & 576 & 6270 & 0.163 & 0.129 & $1.32(1.12-1.56)$ & 10.9 & 0.00095 \\
\hline IIM without CADM & 543 & 6270 & 0.151 & 0.129 & $1.20(1.01-1.43)$ & 4.3 & 0.037 \\
\hline PM & 236 & 6270 & 0.157 & 0.129 & $1.26(0.98-1.62)$ & 3.2 & 0.076 \\
\hline DM total & 340 & 6270 & 0.168 & 0.129 & $1.36(1.11-1.68)$ & 8.6 & 0.0034 \\
\hline DM without CADM & 307 & 6270 & 0.147 & 0.129 & $1.16(0.92-1.46)$ & 1.6 & 0.20 \\
\hline CADM & 33 & 6270 & 0.364 & 0.129 & $3.87(2.23-6.55)$ & 32.0 & $1.5 \times 10^{-8}$ \\
\hline
\end{tabular}

IIM, idiopathic inflammatory myopathy; CADM, clinically amyopathic dermatomyositis;

DM, dermatomyositis; PM, polymyositis; OR, odds ratio; $\mathrm{Cl}$, confidence interval. 


\section{Figure legends}

Figure 1 Association of the WDFY4 locus with clinically amyopathic dermatomyositis (CADM) and its splicing QTL effect on a truncated WDFY4 isoform. (A) Regional association plot of the WDFY4 locus with CADM in Japanese drawn by LocusZoom. The recombination rate estimate is based on East Asian genotype data from the 1000 Genomes project (hg19 coordinates). The dot line indicates the genome-wide significance level $\left(P=5 \times 10^{-8}\right)$. (B) eQTL analysis of the WDFY4 gene and its new exon. RPKM, reads per kilobase per million mapped reads. The line in the middle of the box is the median, and the box edges are the 25 th and 75 th percentiles of 373 samples. (C) Splicing isoform of WDFY4. The RNA-seq data from two Guevadis project samples representing genotypes of rs7919656 were displayed using the Integrative Genomics Viewer (IVG) (top). The new exon extends the exon 36 sequence and introduces a new stop codon (TGA in green). The genomic structure of the reference WDFY4 transcript is shown (bottom). (D) The protein structures of the WDFY4 isoforms. The reference WDFY4 contains a BEACH domain and five WD domains, which are lacking in tr-WDFY. The 11 a.a. sequences at C-terminal of tr-WDFY4 is translated by the new exon. (E) Western blot analysis of V5-tagged. WDFY4 isoforms that were transfected into HEK-293 cells. 
Figure 2 Trans-eQTL analysis of the WDFY4 variant and its application to gene set analysis. (A) Examples of the trans-eQTL effect of the WDFY4 variant (rs7919656). (B) Application of the results of trans-eQTL analysis to wPGSA. We evaluated trans-eQTL effects $(\beta)$ of WDFY4 variant (rs7919656) for distal genes (Gene 1 N) by using wPGSA and estimated the transcriptional factors ( $\mathrm{TF} 1, \mathrm{TF} 2 \ldots)$ responsible for the regulation. (C) Transcriptional regulatory network of TFs responsible for the trans-eQTL effects of WDFY4. The nodes represent the significant TFs identified by wPGSA (FDR $<0.05)$. The circle node represents positive regulation for the trans-eQTL effects and the box node represents negative regulation. Those nodes regulating many other TFs were placed in a higher position and those regulated by many other TFs were placed to the right. The node colors and their density indicate the trans-eQTL effect ( $\beta$ ) of WDFY4 variant for the TF itself (red for positive $\beta$ and blue for negative $\beta$ ). The width of the directed line represents the probability of transcriptional regulation of a TF over other TF gene. (D) NF- $\mathrm{KB}$ activity was evaluated using reporter assays in HEK-293 cells that were transfected with WDFY4 isoforms and were stimulated or not with TNF- $\alpha(1 \mathrm{ng} / \mathrm{ml}$ for 2 hours). Means and s.d. are shown for six technical replicates. The experiment was independently repeated three times, and the representative data were shown. ${ }^{*} P<0.05$ by ANOVA with post-hoc Tukey's test. 


\section{Figure 3. WDFY4 isoforms interact with pattern recognition receptors (PRR) and}

augment their signals. (A) Each transfected PRR signal was evaluated by NF- $\kappa$ B reporter assays of HEK-293 cells co-transfected with each WDFY4 isoform. UNC93B1 was co-transfected in all the experiments for TLR7 and TLR9. Black bars indicate the stimulation reagents used (Poly (I:C), $10 \mu \mathrm{g} / \mathrm{ml}$; LPS, $1 \mu \mathrm{g} / \mathrm{ml}$; Imiquimod, $1 \mu \mathrm{g} / \mathrm{ml}$; ODN2006, $10 \mu \mathrm{g} / \mathrm{ml}$; high molecular weight poly (I:C), $1 \mu \mathrm{g} / \mathrm{ml})$. Means and s.d. are shown for four technical replicates. Each experiment was independently repeated three times, and the representative data were shown. ${ }^{*} P<0.05$ by ANOVA with post-hoc Tukey's test. (B) Immunoprecipitation and western blotting analysis of the HEK-293 cells. Each PRR was immunoprecipitated with anti-FLAG Ab. Co-immunoprecipitated WDFY4 isoforms were then detected by western blotting (WB) using anti-V5 Ab. Each experiment was repeated twice, and the representative data were shown. 
Figure 4 Functional analysis of WDFY4 isoforms as evaluated by apoptosis assays and IRF3 reporter assays. (A) Expression of MDA5 was upregulated by stimulation of HepG2 cells with HMW poly (I:C) for $24 \mathrm{~h}$. The MDA5 transcript was quantified by TaqMan PCR using GAPDH expression as an internal control. (B) tr-WDFY4 enhanced apoptosis in HepG2 cells. Apoptotic cells were determined by assay of caspase activity using flow cytometry. Means and s.d. are shown for four technical replicates. ${ }^{*} P<0.05$ by ANOVA with post-hoc Tukey's test. (C) Induction of Type I interferon in HEK-293 cells that were transfected with WDFY4 isoforms and MDA5 (or TLR3) was evaluated using IRF3 reporter assays. Black bars indicate the stimulation reagents used (Poly (I:C), $10 \mu \mathrm{g} / \mathrm{ml}$; high molecular weight poly (I:C), $1 \mu \mathrm{g} / \mathrm{ml})$. Means and s.d. are shown for four technical replicates. ${ }^{*} P<0.05$ by ANOVA with post-hoc Tukey's test. 
Figure 5 Interaction of MDA5 and tr-WDFY4. (A) Cellular localization of transfected V5-tagged WDFY4 isoforms and FLAG-tagged MDA5 was evaluated by immunofluorescence staining DAPI was used for counter staining of nuclei. Scale bars: $10 \mu \mathrm{m} .100 \mathrm{X}$ objective lens was used. (B) Immunoprecipitation (IP) analysis using sera from CADM patients. MDA5 and tr-WDFY4 were co-transfected into HEK-293 cells, and the cell lysates were immunoprecipitated with sera from CADM patients (three anti-MDA5 positive patients and an anti-MDA5 negative patient. For comparison to another subset of myositis, sera from an anti-aminoacyl tRNA synthetase (ARS) positive DM patient as well as a healthy control (HC) were evaluated. Immunoprecipitated MDA5 proteins were detected by western blotting (WB) with anti-FLAG Ab (upper panel), and co-immunoprecipitated tr-WDFY4 proteins were detected with anti-His $\mathrm{Ab}$ (lower panel). 


\section{(Splicing variant of WDFY4 augments MDA5 signaling and the risk of clinically amyopathic dermatomyositis}

\section{Authors}

Yuta Kochi ${ }^{1}$, Yoichiro Kamatani ${ }^{2}$, Yuya Kondo ${ }^{3}$, Akari Suzuki ${ }^{1}$, Eiryo Kawakami ${ }^{4}$, Ryosuke Hiwa, Yukihide Momozawa ${ }^{6}$, Manabu Fujimoto ${ }^{7,8}$, Masatoshi Jinnin ${ }^{9}$, Yoshiya Tanaka ${ }^{10}$, Takashi Kanda ${ }^{11}$, Robert G. Cooper ${ }^{12,13}$, Hector Chinoy ${ }^{14,15}$, Simon Rothwell ${ }^{15}$, Janine A. Lamb ${ }^{13}$, Jiř́i Vencovský ${ }^{16}$, Heřman Mann ${ }^{16}$, Koichiro Ohmura ${ }^{5}$, Keiko Myouzen ${ }^{1}$, Kazuyoshi Ishigaki ${ }^{2}$, Ran Nakashima ${ }^{5}$, Yuji Hosono ${ }^{5}$, Hiroto Tsuboi ${ }^{3}$, Hidenaga Kawasumi ${ }^{17}$, Yukiko Iwasaki ${ }^{18}$, Hiroshi Kajiyama ${ }^{19}$, Tetsuya Horita ${ }^{20}$, Mariko Ogawa-Momohara $^{21}$, Akito Takamura ${ }^{22}$, Shinichiro Tsunoda ${ }^{23}$, Jun Shimizu ${ }^{24}$, Keishi Fujio $^{18}$, Hirofumi Amano ${ }^{25}$, Akio Mimori ${ }^{26}$, Atsushi Kawakami ${ }^{27}$, Hisanori Umehara ${ }^{28}$, Tsutomu Takeuchi ${ }^{29}$, Hajime Sano ${ }^{23}$, Yoshinao Muro ${ }^{21}$, Tatsuya Atsumi ${ }^{20}$, Toshihide Mimura $^{19}$, Yasushi Kawaguchi ${ }^{17}$, Tsuneyo Mimori ${ }^{5}$, Atsushi Takahashi ${ }^{2}$, Michiaki $\mathrm{Kubo}^{6}$, Hitoshi Kohsaka ${ }^{22}$, Takayuki Sumida ${ }^{3}$, Kazuhiko Yamamoto ${ }^{1,18}$

\section{Corresponding to}

Yuta Kochi, MD, PhD.

Laboratory for Autoimmune Diseases, RIKEN Center for Integrative Medical Sciences 1-7-22 Suehiro-Cho, Tsurumi-Ku, Yokohama, 230-0045, Japan

yuta.kochi@riken.jp

\footnotetext{
Affiliations

${ }^{1}$ Laboratory for Autoimmune Diseases, RIKEN Center for Integrative Medical Sciences, Yokohama, Japan.

${ }^{2}$ Laboratory for Statistical Analysis, RIKEN Center for Integrative Medical Sciences, Yokohama, Japan.

${ }^{3}$ Department of Internal Medicine, Faculty of Medicine, University of Tsukuba, Tsukuba, Japan.

${ }^{4}$ Laboratory for Disease Systems Modeling, RIKEN Center for Integrated Medical Sciences, Yokohama, Japan.

${ }^{5}$ Department of Rheumatology and Clinical Immunology, Kyoto University Graduate School of Medicine, Kyoto, Japan.

${ }^{6}$ Laboratory for Genotyping Development, RIKEN Center for Integrative Medical Sciences, Yokohama, Japan.

${ }^{7}$ Department of Dermatology, Faculty of Medicine, Institute of Medical, Pharmaceutical and Health Sciences, Kanazawa University, Kanazawa, Japan.

${ }^{8}$ Department of Dermatology, University of Tsukuba, Ibaraki, Japan.

${ }^{9}$ Department of Dermatology and Plastic Surgery, Faculty of Life Sciences, Kumamoto University, Kumamoto, Japan.
} 
${ }^{10}$ The First Department of Internal Medicine, University of Occupational and Environmental Health, Kitakyushu, Japan.

${ }^{11}$ Department of Neurology and Clinical Neuroscience, Yamaguchi University Graduate School of Medicine, Ube, Japan.

${ }^{12}$ MRC-ARUK Institute for Ageing and Chronic Disease, University of Liverpool, Liverpool, UK.

${ }^{13}$ Centre for Integrated Genomic Medical Research, Division of Population Health, Health Services Research and Primary Care, School of Health Sciences, Faculty of Biology, Medicine and Health, Manchester Academic Health Science Centre, The University of Manchester, Manchester, UK.

${ }^{14}$ Rheumatology Department, Manchester Academic Health Science Centre, Salford Royal NHS Foundation Trust, Salford, UK.

${ }^{15}$ The National Institute for Health Research Manchester Musculoskeletal Biomedical Research Unit, Central Manchester University Hospitals NHS Foundation Trust, Manchester Academic Health Science Centre, The University of Manchester, Manchester, UK.

${ }^{16}$ Institute of Rheumatology, Charles University, Prague, Czech Republic.

${ }^{17}$ Institute of Rheumatology, Tokyo Women's Medical University, Tokyo, Japan.

${ }^{18}$ Department of Allergy and Rheumatology, Graduate School of Medicine, the University of Tokyo, Tokyo, Japan.

${ }^{19}$ Department of Rheumatology and Applied Immunology, Faculty of Medicine, Saitama Medical University, Saitama, Japan.

${ }^{20}$ Department of Rheumatology, Endocrinology and Nephrology, Faculty of Medicine and Graduate School of Medicine, Hokkaido University, Sapporo, Japan.

${ }^{21}$ Department of Dermatology, Nagoya University Graduate School of Medicine, Nagoya, Japan.

${ }^{22}$ Department of Rheumatology, Graduate School of Medical and Dental Sciences, Tokyo Medical and Dental University, Tokyo, Japan.

${ }^{23}$ Division of Rheumatology Department of Internal Medicine, Hyogo College of Medicine, Hyogo, Japan.

${ }^{24}$ Department of Neurology, Graduate School of Medicine, The University of Tokyo, Japan.

${ }^{25}$ Department of Internal Medicine and Rheumatology, Juntendo University School of Medicine, Tokyo, Japan.

${ }^{26}$ Division of Rheumatic Diseases, National Center for Global Health and Medicine, Tokyo, Japan.

${ }^{27}$ Department of Immunology and Rheumatology, Unit of Advanced Preventive Medical Sciences, Nagasaki University Graduate School of Biomedical Sciences, Nagasaki, Japan.

${ }^{28}$ Department of Hematology and Immunology, Kanazawa Medical University, Ishikawa, Japan

${ }^{29}$ Division of Rheumatology, Department of Internal Medicine, Keio University School of Medicine, Tokyo, Japan. 

important roles. To identify genetic factors of IIM including polymyositis (PM), dermatomyositis (DM), and clinically amyopathic DM (CADM), we performed the first genome-wide association study (GWAS) for IIM in an Asian population.

Methods We genotyped and tested 496,819 SNPs for association using 576 IIM patients and 6,270 control subjects. We also examined the causal mechanism of disease associated variants by in silico analyses using publicly available data sets as well as by in in vitro analyses using reporter assays and apoptosis assays.

Results We identified a variant in WDFY4 that was significantly associated with CADM (rs7919656; odds ratio $\left.(\mathrm{OR})=3.87 ; P=1.5 \times 10^{-8}\right)$. This variant had a cis-splicing quantitative trait locus (QTL) effect for a truncated WDFY4 isoform (tr-WDFY4), with higher expression in the risk allele. Trans-expression QTL (eQTL) analysis of this variant showed a positive correlation with the expression of NF- $\kappa \mathrm{B}$ associated genes. Furthermore, we demonstrated that both WDFY4 and tr-WDFY4 interacted with pattern recognition receptors such as TLR3, TLR4, TLR9, and MDA5 and augmented the $\mathrm{NF}-\kappa \mathrm{B}$ activation by these receptors. WDFY4 isoforms also enhanced MDA5-induced apoptosis, to a greater extent in the tr-WDFY4-transfected cells.

Conclusions As CADM is characterized by the appearance of anti-MDA5 autoantibodies and severe lung inflammation, the WDFY4 variant may play a critical role in the pathogenesis of CADM. 


\section{INTRODUCTION}

Idiopathic inflammatory myopathies (IIM) are autoimmune conditions characterized by muscle weakness and inflammation, and the most common types are polymyositis (PM) and dermatomyositis (DM). ${ }^{12}$ Clinically amyopathic DM (CADM), a subset of DM, lacks distinct muscle features but often manifests rapidly progressive interstitial pneumonia (RPIP). ${ }^{3-6}$ The mortality rate of CADM at 6 months from diagnosis reaches approximately $50 \%$, making it one of the most fatal autoimmune diseases. ${ }^{78}$ The appearance of autoantibodies such as anti-MDA5-antibodies in CADM suggests a role for autoimmunity in the pathogenesis. ${ }^{9-11}$ Although the etiology of IIM remains unknown, virus infection may be the major environmental factor. ${ }^{12}$ In addition, IIM patients and their close relatives are more likely to develop other autoimmune diseases, suggesting shared genetic factors. ${ }^{13-15}$ In fact, a previous genome-wide association studies (GWAS) and candidate gene analyses in European populations demonstrated multiple loci that were shared with other autoimmune diseases. ${ }^{15-18}$

However, when compared with other autoimmune diseases, little is known about the genetic background of IIM. This may be due to the rarity of disease, precluding researchers from designing sufficiently powered studies. In addition, because IIMs are a heterogenous group of rare disorders, an overall pooled analysis might also reduce the power for detecting loci specific to certain subtypes. Therefore, as suggested ${ }^{19}$, studies of disease subsets are needed to increase the power for detecting subset-specific genetic components. Here, to elucidate genetic loci associated with IIM, we performed a GWAS for IIM in the Japanese population. This study was the first GWAS for IIM in an Asian population. We also performed a GWAS for CADM as a subset analysis, which was the first such study in the world. 


\section{Methods}

\section{Subjects}

We enrolled 592 IIM cases (mean age, 55.5 \pm 14.2 years; female, 74.6\%) from 18 medical institutes in Japan. PM/DM fulfilled the definite or probable diagnostic criteria of Bohan and Peter. ${ }^{1}$ The diagnosis of CADM was based on the criteria by Sontheimer et al. ${ }^{36}$ We enrolled 6270 control subjects without history of autoimmune diseases (mean age, $57.0 \pm 13.8$ years; female, $48.2 \%$ ) through the Biobank project ${ }^{20}$. The European subjects (21 CADM cases) were recruited as part of an international myositis genetics consortium (MYOGEN), ${ }^{17}$ and four controls for each case (84 controls) were matched for ethnicity using principal components analysis (PCA) coordinates. ${ }^{21}$ All subjects were provided with written informed consent for participation in the study as approved by the ethical committee of each institutional review board.

\section{GWAS}

We genotyped case samples using HumanOmniExpressExome BeadChip and control samples using HumanOmniExpress BeadChip and HumanExome BeadChip (Illumina). For quality control, we excluded single nucleotide polymorphisms (SNPs) with a call rate $<0.99$, a mean allele frequency $(\mathrm{MAF})<0.05$, and those not in Hardy-Weinberg equilibrium in controls $\left(P<1 \times 10^{-6}\right)$. We removed samples that showed second-degree relatedness or closer by an identity-by-state method. We performed PCA using four reference populations from HapMap data including CEU, YRI, JPT and CHB using SmartPCA software. ${ }^{22}$ We identified outliers not belonging to the JPT/CHB cluster by using the top two principal components. After this screening, we selected 576 cases and 6270 controls for subsequent analysis. This sample size had statistical power $>0.5$ for 
detecting common variants with moderate effects $(\mathrm{MAF}=0.5$ and $\mathrm{OR}=1.5)$ as calculated by QUANTO. ${ }^{23}$ The European samples were genotyped by the Immunochip (Illumina) ${ }^{18}$. We performed genotype imputation within the WDFY4 locus by Minimac ${ }^{24}$ using the 1000 Genomes Project Asian data as reference.

\section{eQTL analysis}

Both cis- and trans- eQTL analyses were performed using Matrix eQTL with the linear model. ${ }^{25}$ We used the Geuvadis project data ${ }^{26}$ of European samples $(n=373)$, “GD462.GeneQuantRPKM.50FN.samplename.resk10.norm.txt” for the gene level eQTL analysis and “GD462.ExonQuantCount.45N.50FN.samplename.resk10.txt” for the exon level eQTL analysis.

\section{WPGSA}

To predict the activities of transcriptional regulators responsible for observed gene expression changes resulting from trans-eQTL effects of tr-WDFY4, we used weighted parametric gene set analysis (wPGSA) ${ }^{27}$ with some modifications (supplementary figure S7). ChIP-seq data of multiple types of human cells were obtained from the GEO-database. In total, 3082 ChIP-seq data of 431 TFs were used. We used the trans-eQTL effects of WDFY4 variant (rs 7919656) on every gene ( $\beta$ obtained by Matrix-eQTL) as input, instead of the log-fold change of the gene expression.

\section{Reporter assay}

The coding sequences of WDFY4 and tr-WDFY4 were cloned into the pcDNA3.1D/V5-His-TOPO vector using cDNAs from EBV-immortalized B cells. 
Human pattern recognition receptors were cloned into the pEF-BOS-FLAG vector (TLR3 was provided by Dr. Matsumoto, Hokkaido University; TLR4 and TLR9 were provided by Dr. Miyake, the University of Tokyo). UNC93B1 was cloned into the pcDNA5/FRT/V5-His vector. UNC93B1 was co-transfected for the experiments of TLR7 or TLR9, because it is essential to deliver these receptors to endolysosomes. ${ }^{28} \mathrm{We}$ cultured HEK-293 cells in DMEM supplemented with 10\% fetal bovine serum. We

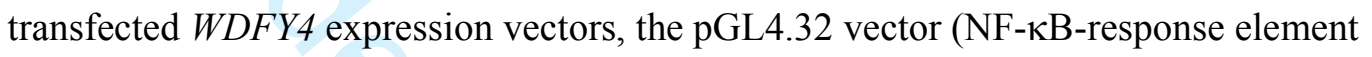
and luc2P) and the pGL4.74 vector (hRluc/TK) (Promega) into cells using the X-tremeGENE HP Transfection Reagent (Roche). The p55C1B-Luc vector ${ }^{29}$ (provided $^{2}$ by Dr. Fujita, Kyoto University) was used instead of pGL4.32 for IRF3 reporter assays. After 18h incubation, the cells were stimulated with poly (I:C) (Enzo Life Sciences), LPS (Sigma-Aldrich), imiquimod (InvivoGen), ODN2006 (InvivoGen), or high molecular weight poly (I:C) ${ }^{30}$ (provided by Dr. Fujita) for $4 \mathrm{~h}$. We then collected the cells and measured luciferase activity using Dual-Luciferase Reporter Assays according to the manufacturer's protocol (Promega).

\section{Immunoprecipitation and western blotting}

HEK-293 cells were transfected with each WDFY4 isoform and PRR and cultured for 24h. The cells were lysed in RIPA buffer ${ }^{31}$ and then incubated with Dynabeads protein-G (Life Technologies) coated with anti-FLAG-antibody (Sigma-Aldrich) and incubated at $4^{\circ} \mathrm{C}$ for $30 \mathrm{~min}$. The beads were then washed and boiled in SDS buffer before electrophoresis through NuPAGE Tris-Acetate gels. The proteins were transferred to PDVF membranes (Millipore), which were then probed with anti-V5-antibody (Invitrogen), visualized using ECL reagent (GE Healthcare), and 
detected using LAS-3000 (Fuji Film). We also performed immunoprecipitation using the sera from patients, instead of anti-FLAG-antibody.

\section{Apoptosis assay}

HepG2 Cells were transfected with each WDFY4 isoform using the Lipofectamine 3000 reagent (Invitrogen). The same vector with cloned $L a c Z$ was also transfected as a mock. Cells were incubated for $24 \mathrm{~h}$ and stimulated with $5 \mu \mathrm{g}$ of poly (I:C). The cells were permeabilized and stained with CaspACE FITC-VAD-FMK (Promega) and anti-His-Alexa 647. Caspase activity was evaluated in the His-positive cells using the BD Accuri C6 flow cytometer (BD Biosciences) to detect apoptotic cells.

\section{Immunofluorescence staining}

HEK-293 cells were grown on coverslips and transfected with the vectors for WDFY4 isoforms and $M D A 5$. Cells were fixed in 4\% paraformaldehyde, permeabilized in $0.25 \%$ Triton X-100, and stained with anti-V5 and anti-mouse IgG-Alexa488 (Invitrogen) for WDFY4 and anti-FLAG-Cy3 (Sigma-Aldrich) for MDA5 detection. DAPI was used for counter-staining. Images were acquired with the confocal microscope N-SIM (Nikon) using $100 \mathrm{X}$ objective lens. The data were analyzed in 3D-SIM mode by using NIS-Elements software (Nikon).

\section{Measurement of anti-MDA5}

We measured anti-MDA5 autoantibodies in the sera from the CADM patients (18 sera were available) by protein-immunoprecipitation. ${ }^{9}$ 


\section{Statistics}

We tested the association of SNPs by allelic test using Plink $1.9,{ }^{32}$ setting the genome-wide significance threshold at $\alpha=5 \times 10^{-8}$. We used a quantile-quantile plot to evaluate the potential effects of population stratification. We used analysis of variance (ANOVA) with post-hoc Tukey's test in the reporter assays and apoptosis assays. 


\section{RESULTS \\ GWAS for IIM}

We genotyped the Japanese samples using Illumina SNP arrays for over 960,000 SNPs. After quality control filtering, the effective sample size was 576 IIM cases and 6,270 controls, and 496,819 SNPs were tested. The genomic inflation factor $\lambda_{G C}$ was 1.024 (supplementary figure S1), suggesting a negligible effect of population stratification. Cases comprised $236 \mathrm{PM}$ and $340 \mathrm{DM}$ patients including $33 \mathrm{CADM}$ patients, and GWASs for these disease subsets were also performed. No significant association signal $\left(P<5 \times 10^{-8}\right)$ was found in any of total IIM, PM or DM (supplementary figure S2). However, when CADM patients were evaluated alone, a significant association was observed for an intronic SNP of WDFY4 (WDFY Family Member 4; rs7919656; $\left.\mathrm{OR}=3.87 ; 95 \% \mathrm{CI}=2.23-6.55 ; P=1.5 \times 10^{-8}\right)($ figure $1 \mathrm{~A}$ and table 1$)$. This SNP showed a nominal association with IIM, even when CADM patients were excluded $(P=0.037)$. In European CADM patients, although no obvious association signal was observed in rs7919656, distinct association signals $\left(r^{2}<0.2\right.$ with $r$ 7919656) were observed for the neighboring variants (rs11101462, $P=0.0092$; rs2889697, $P=0.0058$, supplementary figure S3)

\section{Splicing QTL effect of WDFY4 variant on truncated isoform}

To unravel the causal mechanism of the WDFY4 locus in this disease subset, we first searched for candidate functional variants. Among the variants in strong linkage disequilibrium (LD) with rs7919656 $\left(r^{2}>0.8\right)$ in the 1000 genome project Asian samples, there is no coding variant that altered the amino-acid sequences of neighboring genes (supplementary figure S4). We next searched the expression quantitative trait locus 
(eQTL) data of lymphoblastoid B-cells provided by the Geuvadis project. ${ }^{26}$ When correlations of the SNP (rs7919656) genotype and expression of both the gene and exons were evaluated, a significant eQTL effect for an alternative exon of WDFY4 was found (figure 1B). As no obvious eQTL effect was observed for expression of the whole gene or of neighboring exons (supplementary figure S5) this variant or variants in LD may have a splicing QTL effect for this WDFY4 isoform. Among such variants, two SNPs in intron 35 (rs10776647/rs10776648; figure 1C and supplementary figure S4) were candidate causal variants in this region; however, they did not disrupt classical splicing associated motifs. The alternative exon extends exon 36 of WDFY4, which introduces an alternate stop codon and truncates the protein (figure $1 \mathrm{C}, \mathrm{D})$ ). We verified the existence of this truncated isoform (tr-WDFY4) by cloning it with cDNA from EBV-immortalized B cells and expressing it in HEK-293 cells (figure 1E).

\section{Trans eQTL effect of WDFY4 variant on NF-kB activity}

WDFY4 encodes WDFY family member 4, which has a predicted BEACH domain and five WD domains at the C-terminal $\operatorname{side}^{33}$ (figure 1D). Although WDFY4 is expressed in dendritic cells, neutrophils, B-cells and macrophages (supplementary figure S6), to our knowledge, there have been no studies into its protein function. To obtain insight regarding the function of $W D F Y 4$, we performed trans-eQTL analysis of the variant using the same expression data of lymphoblastoid B-cells. A total of 387 genes were correlated with the number of risk alleles (uncorrected $P<0.05$ ), although no gene displayed genome-wide significant correlation (FDR $<0.05$ ). Notably, 279 genes (72.1\%) were positively correlated, indicating that upregulated expression of tr-WDFY4 results in upregulation of these genes. Among the top 10 genes with the largest 
trans-eQTL effects ( $\beta$ as calculated in the Matrix eQTL analysis), TYK2 and PTPN6 are well known regulators of signal pathways in B-cells (figure 2A). We then sought to identify the transcriptional factors (TFs) present downstream of tr-WDFY4 by weighted parametric gene set analysis (wPGSA). ${ }^{27}$ wPGSA utilizes all available ChIP-seq data obtained from multiple types of human cells and predicts the activities of transcriptional regulators responsible for observed gene expression changes (figure $2 \mathrm{~B}$ and supplementary figure S7). When trans-eQTL effects $(\beta)$ were used as input, among the $431 \mathrm{TFs}$ evaluated, $6 \mathrm{TFs}$ displayed significant associations ( $\mathrm{FDR}<0.05$ ) (figure $2 \mathrm{C}$ and supplementary table S1). We focused on TF65 (RELA/p65), because it comprises nuclear factor-kappa $\mathrm{B}(\mathrm{NF}-\mathrm{\kappa B})$ heterodimers that are one of the most important TFs in the immune system. ${ }^{34}$ Based on these findings, we assumed that the NF- $\kappa$ B pathway is activated downstream of WDFY4. Indeed, NF- $\kappa B$ reporter assays showed that both WDFY4 and tr-WDFY4 enhanced NF- $\kappa B$ activity when transfected into HEK-293 cells with stimulation of TNF- $\alpha$ (figure 2D).

\section{WDFY4 augments signals of innate pattern recognition receptors}

These observations, as well as a recent report that WDFY1, another member of the WDFY gene family, augmented TLR3 and TLR9 signaling ${ }^{35}$, motivated us to investigate the role of WDFY4 in PRR signaling. We chose TLR3/TLR4/TLR7/TLR9 and MDA5 for analysis considering their implied roles in disease pathogenesis ${ }^{4637}$, as well as their potential to activate NF- $\kappa \mathrm{B} .{ }^{38}$ We first performed NF- $\kappa \mathrm{B}$ reporter assays by simultaneously transfecting each WDFY4 isoform with each PRR. While both

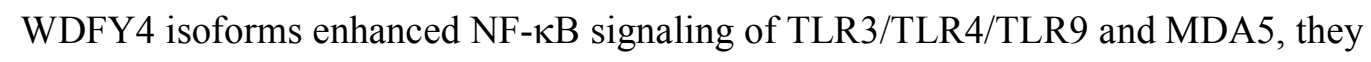
had a negligible effect on TLR7 signaling (figure 3A). Of note, tr-WDFY4 markedly 
enhanced the MDA5 signal when stimulated with poly (I:C), a synthetic analog of double-stranded RNA. We also examined the impact of WDFY4 isoforms on cytokine production by quantifying the levels of IL-8 in the cell culture supernatants. Although both isoforms significantly increased the IL-8 production in steady state, the difference to mock transfectant became insignificant when cells were stimulated with poly (I:C) (supplementary figure S8). Next, we examined the physical interaction of WDFY4 isoforms and these PRRs by co-immunoprecipitation. Both V5-tagged WDFY4 isoforms interacted with these FLAG-tagged PRRs to greater or lesser degrees (figure 3B). The strongest interaction was observed between tr-WDFY4 and MDA5, which was consistent with the findings of reporter assays.

MDA5-mediated sensing of virus triggers not only endogenous immune responses but also cellular apoptosis to eliminate virus-infected cells. ${ }^{39}$ Therefore, to further evaluate the function of WDFY4 in the context of MDA5 signaling, we performed apoptosis assays. We used HepG2 cells for these assays, because stimulation of these cells with poly $(\mathrm{I}: \mathrm{C})$ is accompanied by upregulation of MDA5 and results in the induction of apoptosis $^{40}$ (figure 4A). When HepG2 cells were transfected with the WDFY4 isoforms and stimulated with/without poly (I:C), apoptosis was augmented to a greater extent in the tr-WDFY4-transfected cells than in the WDFY4-transfected cells (figure 4B). Again, this result is consistent with the observations regarding these isoforms in the NF- $\mathrm{kB}$ assays. As the apoptosis induced by MDA5 was reported to be independent of type I interferon signals ${ }^{41}$, we evaluated the effect of WDFY4 isoforms on type I interferon induction by co-transfected MDA5 using IRF3 reporter assays ${ }^{29}$. For comparison, we also examined TLR3 signaling. While no effect of WDFY4 isoforms on TLR3 induction of interferon was detected, MDA5 induction of interferon was markedly 
repressed when the WDFY4 isoforms were co-transfected with MDA5 (figure 4C).

These findings suggested that the WDFY4 isoforms are able to interact with MDA5 and alter its signaling in cells. This possibility was further supported by the observations that transfected WDFY4 isoforms and MDA5 co-localized in the cytoplasm of cells as assessed using immunofluorescence (figure 5A), and that the anti-MDA5-positive sera from patients could co-immunoprecipitate tr-WDFY4 proteins (figure 5B). 


\section{DISCUSSION}

We here demonstrated a significant association between a variant in WDFY4 and CADM susceptibility. Association of WDFY4 locus has been previously reported with systemic lupus erythematosus (SLE) ${ }^{42}$ and rheumatoid arthritis. ${ }^{43}$ A recent study reported that the SLE risk variant (rs2663052) also had a splicing QTL effect on WDFY4. ${ }^{44}$ However, rs2663052 is not in tight LD with the CADM risk variant (rs7919656, $r^{2}=0.46$ in the Asian samples), and rs2663052 displayed a weaker association signal in CADM in the present study $(P=0.0012)$. A missense variant (rs7097397), which is in moderate LD with rs7919656 $\left(r^{2}=0.58\right.$ in the Asian samples), may be another candidate causal variant in $\mathrm{SLE}^{42}$, but the association in CADM was moderate $(P=0.0022)$.

The fact that the CADM risk variant was also associated with upregulated expression of tr-WDFY4 suggested that WDFY4 was a strong candidate gene in this locus.

Although the biological function of WDFY4 was unknown, our analysis by wPGSA utilizing the trans-eQTL effects of $W D F Y 4$ variant implied that $\mathrm{NF}-\kappa \mathrm{B}$ was involved in the downstream regulation of WDFY4. Indeed, as demonstrated by in vitro assays, tr-WDFY4 augmented the NF- $\mathrm{BB}$ signals induced by PRRs including MDA5. Our in vitro assays also demonstrated that the intact WDFY4 augmented MDA5-induced $\mathrm{NF}-\kappa \mathrm{B}$ signal and apoptosis, but to lesser extent than tr-WDFY4. The differences in function between the intact WDFY4 and tr-WDFY4 should be further examined, but the lack of predicted WDFY4 protein domains (BEACH and WD domains) may have a marked impact on tr-WDFY4 function. Moreover, the suppression of IRF3 activity observed in the reporter assays suggests the role of WDFY4 in interferon signaling pathway. Because WDFY4 was shown to be downregulated in SLE patients ${ }^{45}$, WDFY4 
isoforms may control the balance between interferon and NF- $\kappa$ B pathways, which should be further examined.

The lack of an association for rs7919656 (sQTL variant) in the European patients might be due to a lack of statistical power, because it diminished to 0.32 if a moderate effect size $(\mathrm{OR}=1.5)$ was assumed. However, as rs11101462, which displayed a positive association signal in Europeans, had an eQTL effect for WDFY4 in peripheral blood samples with higher expression in the risk allele (supplementary table S2), WDFY4 may also have a role in CADM in Europeans. The genetic heterogeneity observed between these populations might be partially explained by environmental factors. Because the extent of MDA5 signal transduction depends on the characteristic of ligands (viruses) such as their length ${ }^{30}$, the effects of WDFY4 isoforms on the MDA5 signaling pathway may also depend on the ligands. Therefore, if viruses trigger the disease and the type of viruses is different between the populations, the genetic role of the two variants and their related WDFY4 isoforms may also differ. The higher incidence of RPIP reported in Asian populations ${ }^{446}$ may indeed suggest etiological heterogeneity in CADM.

An autoimmune reaction to MDA5 may be a central event in CADM pathogenesis, because CADM is characterized by the appearance of anti-MDA5-Abs. This phenomenon was also observed in $72.2 \%(n=13 / 18)$ of the CADM patients evaluated, and these anti-MDA5-positive sera from patients could co-immunoprecipitate tr-WDFY4 proteins. Interestingly, the serum from an anti-MDA5-negative patient could co-immunoprecipitiate tr-WDFY4 proteins, which might suggest the presence of anti-WDFY4 antibody. An inflammatory condition following exposure to antigens derived from apoptotic cells is one of the main mechanisms that trigger autoimmune disorders as shown in the animal models. ${ }^{47}$ Therefore, our findings that tr-WDFY4 
enhanced both inflammatory signals and apoptosis induced by MDA5 suggest that tr-WDFY4 has a critical role in the autoimmune response to MDA5 and possibly to WDFY4.

Moreover, because tr-WDFY4 augmented the NF- $\mathrm{KB}$ signals induced by PRRs, tr-WDFY4 may be also involved in the pathogenesis of RPIP. However, the high mortality rate of RPIP might have precluded the CADM patients with RPIP from participating in the study. Therefore, our samples might have underestimated the role of WDFY4 variant in this disease subset, particularly in patients with RPIP. To determine whether the WDFY4 variant can be used as a predictive biomarker for RPIP, a prospective study of new-onset CADM patients (or anti-MDA5-positive patients) is needed. Meanwhile, by assessing the environmental exposure, viral infections in particular, the role of gene-environmental interactions can be examined. As RPIP in CADM is still refractory to intense immunosuppressive therapies, tr-WDFY4 would be a candidate target in the treatment of CADM. 


\section{Acknowledgments}

We thank K. Kobayashi and M. Abe for their technical assistance and Dr. R. Yamada and Dr. Y. Okada for their advice at RIKEN. We thank Dr. F. W. Miller at the US National Institutes of Health, who established the MYOGEN consortium, for arranging the collaboration. We thank Dr. K. Miyake at the University of Tokyo, Dr. H. Kato and Dr. T. Fujita at Kyoto University for providing technical advice and reagents.

\section{Funding}

This work was supported by the Health and Labour Sciences Research Grants for research on intractable diseases (The Research Team for Autoimmune Diseases) from the Ministry of Health, Labour and Welfare of Japan. This work was also supported by the BioBank Japan Project of the Ministry of Education, Culture, Sports, Sciences and Technology of the Japanese government.

\section{Contributors}

Y.Kochi designed the study and drafted the manuscript. T.S., H.K., and Y.K. directed the Research Team for Autoimmune Diseases and revised the manuscript. Y.Momozawa. and M.K. performed the genotyping for GWAS. Y.Kochi, Y.Kamatani, and A.Takahashi analyzed the GWAS data. Y.Kochi, E.K., and K.I. performed eQTL analysis and wPGSA. Y.Kochi and A.S. conducted the reporter assays and immunoprecipitation. K.M. performed apoptosis assays and immunofluorescence staining. R.H., K.O., S.T., and T.M. assayed anti-MDA5 Abs and performed immunoprecipitation analysis using the sera from CADM patients. The following authors contributed to the Research Team for Autoimmune Diseases and its sample collection: Y.Kondo, M.F., M.J., Y.T., T.K., 


\begin{abstract}
R.N., Y.H., H.T., H.Kawasumi, Y.I., H.Kajiyama, T.H., M.O-M., A.Takamura, S.T., J.S., K.F., H.A., A.M., A.K., H.U.,T.T., Y.Muro, T.A., T.Mimura, Y.Kawaguchi, T.Mimori, M.K., H.Kohsaka, T.S., and K.Y. M.K. directed the analysis of the BioBank samples.

R.G.C., H.C., J.V., and H.M. collected the European samples. S.R. and J.A.L. analyzed European CADM data.
\end{abstract}

\title{
Conflict of interest
}

The authors have declared that no conflict of interest exists. 


\section{REFERENCES}

1 Bohan A, Peter JB. Polymyositis and dermatomyositis (first of two parts). $N$ Engl $J$ Med 1975;292:344-7.

2 Dalakas MC. Inflammatory muscle diseases. N Engl J Med 2015;372:1734-47.

3 Sontheimer RD. Would a new name hasten the acceptance of amyopathic dermatomyositis (dermatomyositis siné myositis) as a distinctive subset within the idiopathic inflammatory dermatomyopathies spectrum of clinical illness? J Am Acad Dermatol 2002;46:626-36.

4 Mimori T, Nakashima R, Hosono Y. Interstitial lung disease in myositis: clinical subsets, biomarkers, and treatment. Current rheumatology reports 2012;14:264-74.

5 Sontheimer RD. MDA5 autoantibody-another indicator of clinical diversity in dermatomyositis. Annals of translational medicine 2017;5:160.

6 Gerami P, Schope JM, McDonald L, et al. A systematic review of adult-onset clinically amyopathic dermatomyositis (dermatomyositis sine myositis): a missing link within the spectrum of the idiopathic inflammatory myopathies. J Am Acad Dermatol 2006;54:597-613.

7 Koga T, Fujikawa K, Horai Y, et al. The diagnostic utility of anti-melanoma differentiation-associated gene 5 antibody testing for predicting the prognosis of Japanese patients with DM. Rheumatology (Oxford) 2012;51:1278-84.

8 Mukae H, Ishimoto H, Sakamoto N, et al. Clinical differences between interstitial lung disease associated with clinically amyopathic dermatomyositis and classic dermatomyositis. Chest 2009;136:1341-7.

9 Nakashima R, Imura Y, Kobayashi S, et al. The RIG-I-like receptor IFIH1/MDA5 is a dermatomyositis-specific autoantigen identified by the anti-CADM-140 antibody. Rheumatology (Oxford) 2010;49:433-40.

10 Senecal JL, Raynauld JP, Troyanov Y. Editorial: A New Classification of Adult Autoimmune Myositis. Arthritis Rheumatol 2017;69:878-84.

11 Satoh M, Tanaka S, Ceribelli A, et al. A Comprehensive Overview on Myositis-Specific Antibodies: New and Old Biomarkers in Idiopathic Inflammatory Myopathy. Clin Rev Allergy Immunol 2017;52:1-19.

12 Gan L, Miller FW. State of the art: what we know about infectious agents and myositis. Curr Opin Rheumatol 2011;23:585-94.

13 Ginn LR, Lin JP, Plotz PH, et al. Familial autoimmunity in pedigrees of idiopathic inflammatory myopathy patients suggests common genetic risk factors for many autoimmune diseases. Arthritis Rheum 1998;41:400-5.

14 Niewold TB, Wu SC, Smith M, et al. Familial aggregation of autoimmune disease in 
juvenile dermatomyositis. Pediatrics 2011;127:e1239-46.

15 Miller FW, Cooper RG, Vencovsky J, et al. Genome-wide association study of dermatomyositis reveals genetic overlap with other autoimmune disorders. Arthritis Rheum 2013;65:3239-47.

Jani M, Massey J, Wedderburn LR, et al. Genotyping of immune-related genetic variants identifies TYK2 as a novel associated locus for idiopathic inflammatory myopathies. Ann Rheum Dis 2014;73:1750-2.

17 Miller FW, Chen W, O'Hanlon TP, et al. Genome-wide association study identifies HLA 8.1 ancestral haplotype alleles as major genetic risk factors for myositis phenotypes. Genes Immun 2015;16:470-80.

18 Rothwell S, Cooper RG, Lundberg IE, et al. Dense genotyping of immune-related loci in idiopathic inflammatory myopathies confirms HLA alleles as the strongest genetic risk factor and suggests different genetic background for major clinical subgroups. Ann Rheum Dis 2016;75:1558-66.

19 Bhattacharjee S, Rajaraman $\mathrm{P}$, Jacobs KB, et al. A subset-based approach improves power and interpretation for the combined analysis of genetic association studies of heterogeneous traits. Am J Hum Genet 2012;90:821-35.

20 Nagai A, Hirata M, Kamatani Y, et al. Overview of the BioBank Japan Project: Study design and profile. J Epidemiol 2017;27:S2-S8.

21 Gregersen PK, Kosoy R, Lee AT, et al. Risk for myasthenia gravis maps to a (151) Pro-->Ala change in TNIP1 and to human leukocyte antigen-B*08. Ann Neurol 2012;72:927-35.

22 Price AL, Patterson NJ, Plenge RM, et al. Principal components analysis corrects for stratification in genome-wide association studies. Nat Genet 2006;38:904-9.

23 Gauderman WJ. Sample size requirements for matched case-control studies of gene-environment interaction. Stat Med 2002;21:35-50.

24 Howie B, Fuchsberger C, Stephens M, et al. Fast and accurate genotype imputation in genome-wide association studies through pre-phasing. Nat Genet 2012;44:955-9.

25 Shabalin AA. Matrix eQTL: ultra fast eQTL analysis via large matrix operations. Bioinformatics 2012;28:1353-8.

26 Lappalainen T, Sammeth M, Friedlander MR, et al. Transcriptome and genome sequencing uncovers functional variation in humans. Nature 2013;501:506-11.

27 Kawakami E, Nakaoka S, Ohta T, et al. Weighted enrichment method for prediction of transcription regulators from transcriptome and global chromatin immunoprecipitation data. Nucleic Acids Res 2016;44:5010-21.

28 Kim YM, Brinkmann MM, Paquet ME, et al. UNC93B1 delivers nucleotide-sensing 
toll-like receptors to endolysosomes. Nature 2008;452:234-8.

29 Devaraj SG, Wang N, Chen Z, et al. Regulation of IRF-3-dependent innate immunity by the papain-like protease domain of the severe acute respiratory syndrome coronavirus. J Biol Chem 2007;282:32208-21.

30 Kato H, Takeuchi O, Mikamo-Satoh E, et al. Length-dependent recognition of double-stranded ribonucleic acids by retinoic acid-inducible gene-I and melanoma differentiation-associated gene 5. J Exp Med 2008;205:1601-10.

31 Kochi Y, Myouzen K, Yamada R, et al. FCRL3, an autoimmune susceptibility gene, has inhibitory potential on B-cell receptor-mediated signaling. $J$ Immunol 2009;183:5502-10.

32 Purcell S, Neale B, Todd-Brown K, et al. PLINK: a tool set for whole-genome association and population-based linkage analyses. Am J Hum Genet 2007;81:559-75.

33 Cullinane AR, Schaffer AA, Huizing M. The BEACH is hot: a LYST of emerging roles for BEACH-domain containing proteins in human disease. Traffic 2013;14:749-66.

34 Vallabhapurapu S, Karin M. Regulation and function of NF-kappaB transcription factors in the immune system. Annu Rev Immunol 2009;27:693-733.

$35 \mathrm{Hu}$ YH, Zhang Y, Jiang LQ, et al. WDFY1 mediates TLR3/4 signaling by recruiting TRIF. EMBO reports 2015;16:447-55.

36 Brunn A, Zornbach K, Hans VH, et al. Toll-like receptors promote inflammation in idiopathic inflammatory myopathies. J Neuropathol Exp Neurol 2012;71:855-67.

37 Haq SA, Tournadre A. Idiopathic inflammatory myopathies: from immunopathogenesis to new therapeutic targets. Int J Rheum Dis 2015;18:818-25.

38 Kawai T, Akira S. Toll-like receptor and RIG-I-like receptor signaling. Ann $N$ Y Acad Sci 2008;1143:1-20.

39 Barral PM, Sarkar D, Su ZZ, et al. Functions of the cytoplasmic RNA sensors RIG-I and MDA-5: key regulators of innate immunity. Pharmacol Ther 2009;124:219-34.

40 Peng S, Geng J, Sun R, et al. Polyinosinic-polycytidylic acid liposome induces human hepatoma cells apoptosis which correlates to the up-regulation of RIG-I like receptors. Cancer Sci 2009;100:529-36.

41 Besch R, Poeck H, Hohenauer T, et al. Proapoptotic signaling induced by RIG-I and MDA- 5 results in type I interferon-independent apoptosis in human melanoma cells. $J$ Clin Invest 2009;119:2399-411.

42 Yang W, Shen N, Ye DQ, et al. Genome-wide association study in Asian populations identifies variants in ETS1 and WDFY4 associated with systemic lupus erythematosus. PLoS genetics 2010;6:e1000841.

43 Okada Y, Wu D, Trynka G, et al. Genetics of rheumatoid arthritis contributes to 
biology and drug discovery. Nature 2014;506:376-81.

44 Odhams CA, Cortini A, Chen L, et al. Mapping eQTLs with RNA-seq reveals novel susceptibility genes, non-coding RNAs and alternative-splicing events in systemic lupus erythematosus. Hum Mol Genet 2017;26:1003-17.

45 Kuijpers TW, Vendelbosch S, Berg M, et al. Killer immunoglobulin receptor genes in spondyloarthritis. Curr Opin Rheumatol 2016;28:368-75.

46 Parronchi P, Radice A, Palterer B, et al. MDA5-positive dermatomyositis: an uncommon entity in Europe with variable clinical presentations. Clin Mol Allergy 2015;13:22.

47 Marshak-Rothstein A, Rifkin IR. Immunologically active autoantigens: the role of toll-like receptors in the development of chronic inflammatory disease. Annu Rev Immunol 2007;25:419-41. 
Table 1 Association analysis of rs7919656 with IIM

\begin{tabular}{|c|c|c|c|c|c|c|c|}
\hline \multirow{2}{*}{ Disease } & \multicolumn{2}{|c|}{ Numbers } & \multicolumn{2}{|c|}{ Allele A frequency } & \multicolumn{3}{|c|}{ Allelic comparison } \\
\hline & Patients & Controls & Patients & Controls & OR ( $95 \% \mathrm{Cl})$ & $\chi^{2}$ & $P$ value \\
\hline IIM total & 576 & 6270 & 0.163 & 0.129 & $1.32(1.12-1.56)$ & 10.9 & 0.00095 \\
\hline IIM without CADM & 543 & 6270 & 0.151 & 0.129 & $1.20(1.01-1.43)$ & 4.3 & 0.037 \\
\hline PM & 236 & 6270 & 0.157 & 0.129 & $1.26(0.98-1.62)$ & 3.2 & 0.076 \\
\hline DM total & 340 & 6270 & 0.168 & 0.129 & $1.36(1.11-1.68)$ & 8.6 & 0.0034 \\
\hline DM without CADM & 307 & 6270 & 0.147 & 0.129 & $1.16(0.92-1.46)$ & 1.6 & 0.20 \\
\hline CADM & 33 & 6270 & 0.364 & 0.129 & $3.87(2.23-6.55)$ & 32.0 & $1.5 \times 10^{-8}$ \\
\hline
\end{tabular}

IIM, idiopathic inflammatory myopathy; CADM, clinically amyopathic dermatomyositis;

DM, dermatomyositis; PM, polymyositis; OR, odds ratio; $\mathrm{Cl}$, confidence interval. 


\section{Figure legends}

Figure 1 Association of the WDFY4 locus with clinically amyopathic dermatomyositis (CADM) and its splicing QTL effect on a truncated WDFY4 isoform. (A) Regional association plot of the WDFY4 locus with CADM in Japanese drawn by LocusZoom. The recombination rate estimate is based on East Asian genotype data from the 1000 Genomes project (hg19 coordinates). The dot line indicates the genome-wide significance level $\left(P=5 \times 10^{-8}\right)$. (B) eQTL analysis of the WDFY4 gene and its new exon. RPKM, reads per kilobase per million mapped reads. The line in the middle of the box is the median, and the box edges are the 25 th and 75 th percentiles of 373 samples. (C) Splicing isoform of WDFY4. The RNA-seq data from two Guevadis project samples representing genotypes of rs7919656 were displayed using the Integrative Genomics Viewer (IVG) (top). The new exon extends the exon 36 sequence and introduces a new stop codon (TGA in green). The genomic structure of the reference WDFY4 transcript is shown (bottom). (D) The protein structures of the WDFY4 isoforms. The reference WDFY4 contains a BEACH domain and five WD domains, which are lacking in tr-WDFY. The 11 a.a. sequences at C-terminal of tr-WDFY4 is translated by the new exon. (E) Western blot analysis of V5-tagged. WDFY4 isoforms that were transfected into HEK-293 cells. 
Figure 2 Trans-eQTL analysis of the WDFY4 variant and its application to gene set analysis. (A) Examples of the trans-eQTL effect of the WDFY4 variant (rs7919656). (B) Application of the results of trans-eQTL analysis to wPGSA. We evaluated trans-eQTL effects $(\beta)$ of WDFY4 variant (rs7919656) for distal genes (Gene 1 N) by using wPGSA and estimated the transcriptional factors ( $\mathrm{TF} 1, \mathrm{TF} 2 \ldots)$ responsible for the regulation. (C) Transcriptional regulatory network of TFs responsible for the trans-eQTL effects of WDFY4. The nodes represent the significant TFs identified by wPGSA (FDR < 0.05). The circle node represents positive regulation for the trans-eQTL effects and the box node represents negative regulation. Those nodes regulating many other TFs were placed in a higher position and those regulated by many other TFs were placed to the right. The node colors and their density indicate the trans-eQTL effect ( $\beta$ ) of WDFY4 variant for the TF itself (red for positive $\beta$ and blue for negative $\beta$ ). The width of the directed line represents the probability of transcriptional regulation of a TF over other TF gene. (D) NF- $\mathrm{BB}$ activity was evaluated using reporter assays in HEK-293 cells that were transfected with WDFY4 isoforms and were stimulated or not with TNF- $\alpha(1 \mathrm{ng} / \mathrm{ml}$ for 2 hours). Means and s.d. are shown for six technical replicates. The experiment was independently repeated three times, and the representative data were shown. ${ }^{*} P<0.05$ by ANOVA with post-hoc Tukey's test. 
Figure 3. WDFY4 isoforms interact with pattern recognition receptors (PRR) and augment their signals. (A) Each transfected PRR signal was evaluated by $\mathrm{NF}^{-} \kappa \mathrm{B}$ reporter assays of HEK-293 cells co-transfected with each WDFY4 isoform. UNC93B1 was co-transfected in all the experiments for TLR7 and TLR9. Black bars indicate the stimulation reagents used (Poly (I:C), $10 \mu \mathrm{g} / \mathrm{ml}$; LPS, $1 \mu \mathrm{g} / \mathrm{ml}$; Imiquimod, $1 \mu \mathrm{g} / \mathrm{ml}$; ODN2006, $10 \mu \mathrm{g} / \mathrm{ml}$; high molecular weight poly (I:C), $1 \mu \mathrm{g} / \mathrm{ml}$ ). Means and s.d. are shown for four technical replicates. Each experiment was independently repeated three times, and the representative data were shown. ${ }^{*} P<0.05$ by ANOVA with post-hoc Tukey's test. (B) Immunoprecipitation and western blotting analysis of the HEK-293 cells. Each PRR was immunoprecipitated with anti-FLAG Ab. Co-immunoprecipitated WDFY4 isoforms were then detected by western blotting (WB) using anti-V5 Ab. Each experiment was repeated twice, and the representative data were shown. 
Figure 4 Functional analysis of WDFY4 isoforms as evaluated by apoptosis assays and IRF3 reporter assays. (A) Expression of MDA5 was upregulated by stimulation of HepG2 cells with HMW poly (I:C) for $24 \mathrm{~h}$. The MDA5 transcript was quantified by TaqMan PCR using GAPDH expression as an internal control. (B) tr-WDFY4 enhanced apoptosis in HepG2 cells. Apoptotic cells were determined by assay of caspase activity using flow cytometry. Means and s.d. are shown for four technical replicates. ${ }^{*} P<0.05$ by ANOVA with post-hoc Tukey's test. (C) Induction of Type I interferon in HEK-293 cells that were transfected with WDFY4 isoforms and MDA5 (or TLR3) was evaluated using IRF3 reporter assays. Black bars indicate the stimulation reagents used (Poly (I:C), $10 \mu \mathrm{g} / \mathrm{ml}$; high molecular weight poly (I:C), $1 \mu \mathrm{g} / \mathrm{ml})$. Means and s.d. are shown for four technical replicates. ${ }^{*} P<0.05$ by ANOVA with post-hoc Tukey's test. 
Figure 5 Interaction of MDA5 and tr-WDFY4. (A) Cellular localization of transfected V5-tagged WDFY4 isoforms and FLAG-tagged MDA5 was evaluated by immunofluorescence staining DAPI was used for counter staining of nuclei. Scale bars: 10 $\mu \mathrm{m}$. 100X objective lens was used. (B) Immunoprecipitation (IP) analysis using sera from CADM patients. MDA5 and tr-WDFY4 were co-transfected into HEK-293 cells, and the cell lysates were immunoprecipitated with sera from CADM patients (three anti-MDA5 positive patients and an anti-MDA5 negative patient. For comparison to another subset of myositis, sera from an anti-aminoacyl tRNA synthetase (ARS) positive DM patient as well as a healthy control (HC) were evaluated. Immunoprecipitated MDA5 proteins were detected by western blotting (WB) with anti-FLAG Ab (upper panel), and co-immunoprecipitated tr-WDFY4 proteins were detected with anti-His $\mathrm{Ab}$ (lower panel). 
A

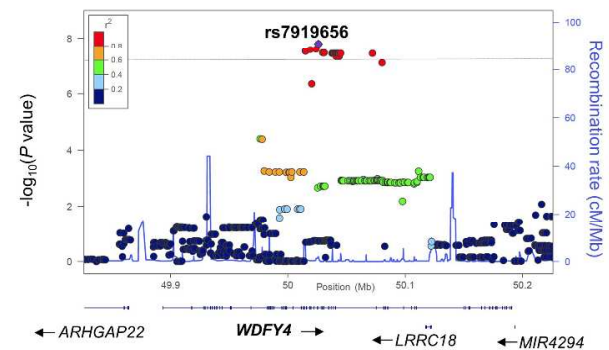

B
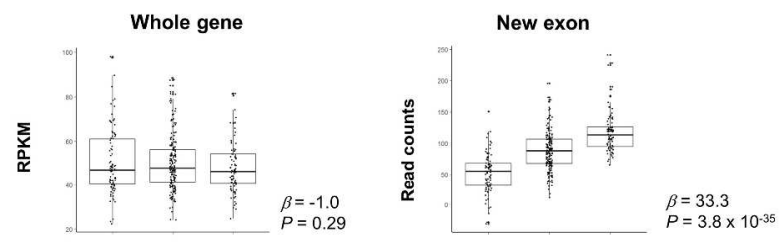

GG GA AA

Genotype of rs7919656 ( $A$ = the risk allele)

C
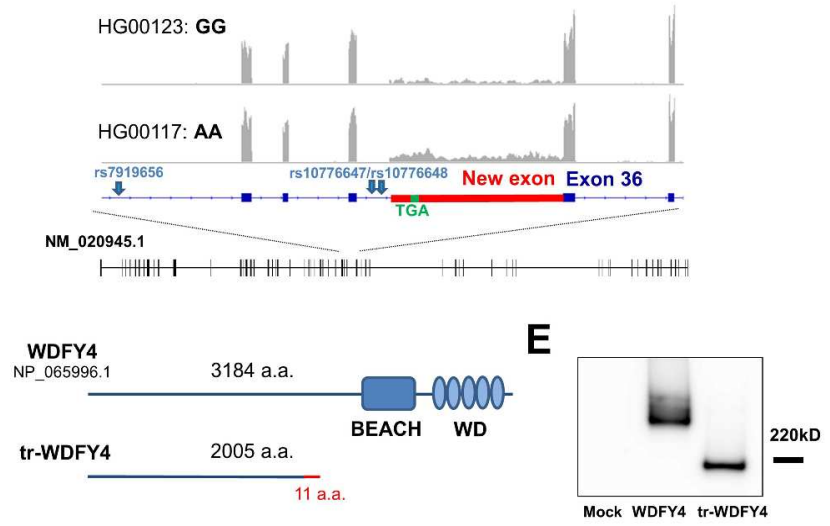

Fig. 1

Fig. 1

$254 \times 381 \mathrm{~mm}(300 \times 300$ DPI $)$

https://mc.manuscriptcentral.com/ard 


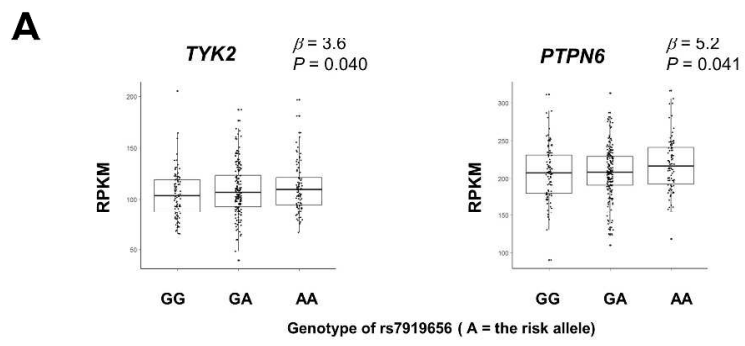

B

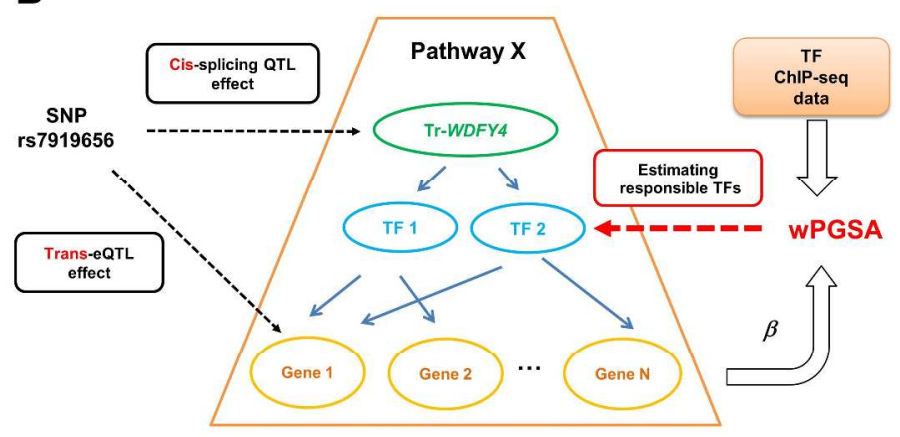

C

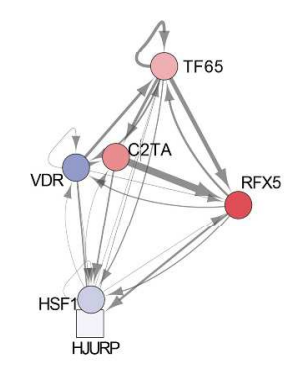

D

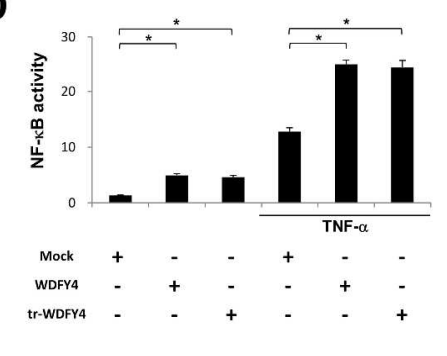

Fig. 2

Fig. 2

$254 \times 381 \mathrm{~mm}(300 \times 300$ DPI $)$ 
A
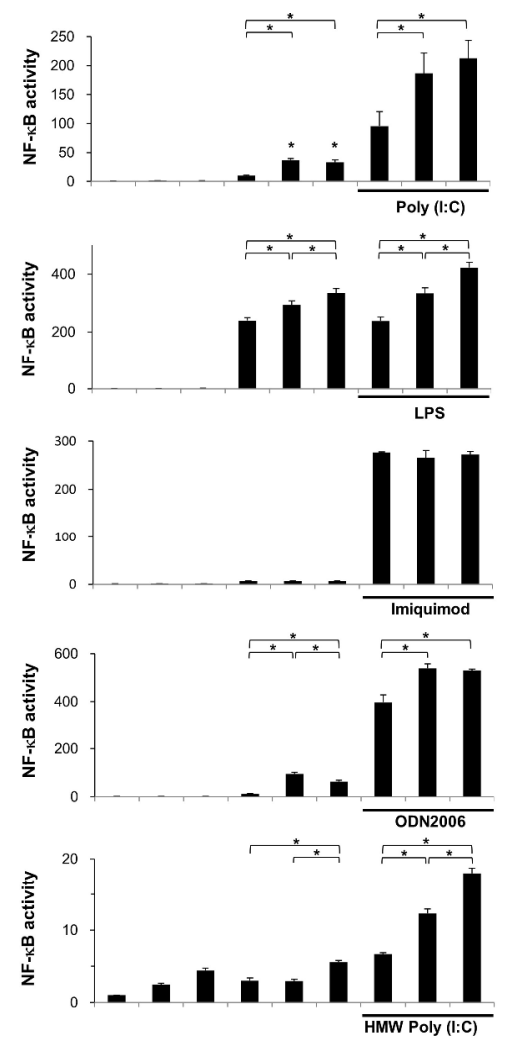

Mock $+-\quad+\quad+-+$

$\begin{array}{llllllllll}\text { WDFY4 } & - & + & - & - & + & - & - & + & - \\ \text { Ir-WDFY4 } & - & - & + & - & - & + & - & - & +\end{array}$

PRR

Fig. 3
TLR9

TLR4

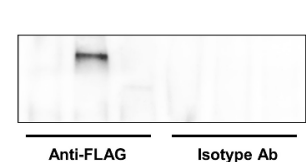

TLR7

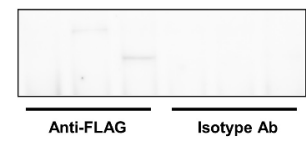

B
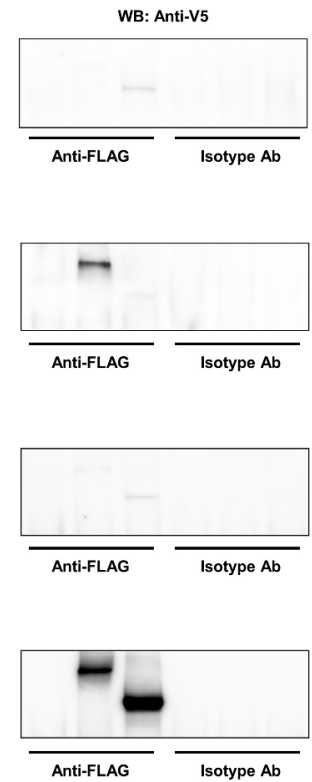

MDA5

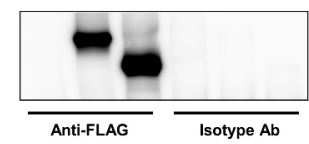

Mock + +

WDFY $4++-++$

WDDFY $-\quad++-+$

Fig. 3

$254 \times 381 \mathrm{~mm}(300 \times 300$ DPI $)$ 


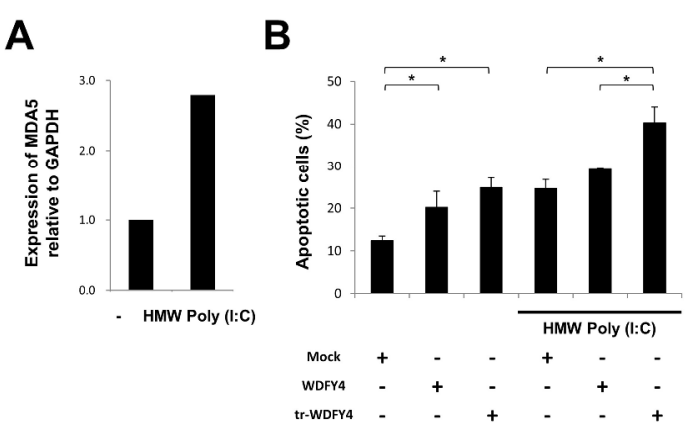

C
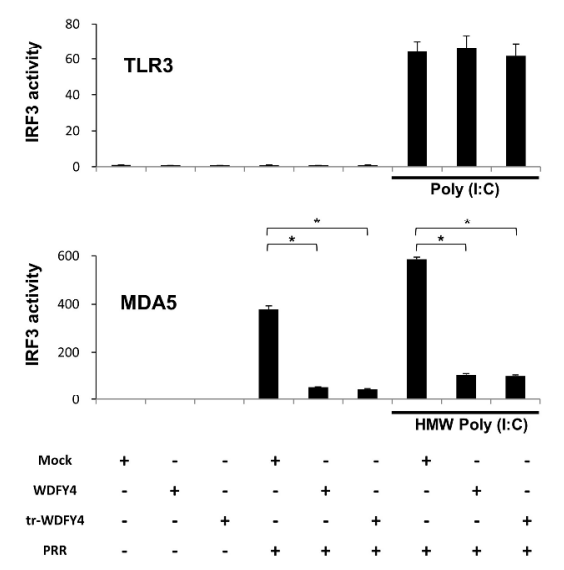

Fig. 4

Fig. 4

$254 \times 381 \mathrm{~mm}(300 \times 300$ DPI $)$ 
A

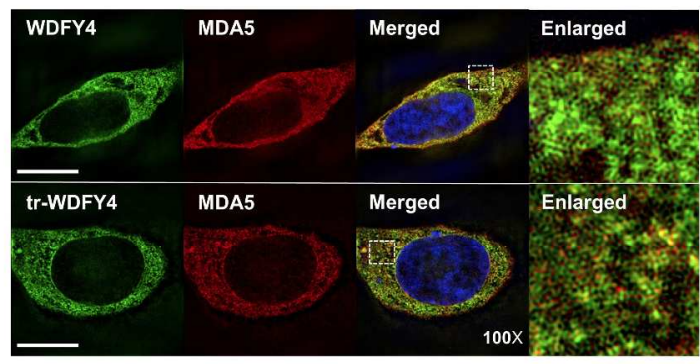

B

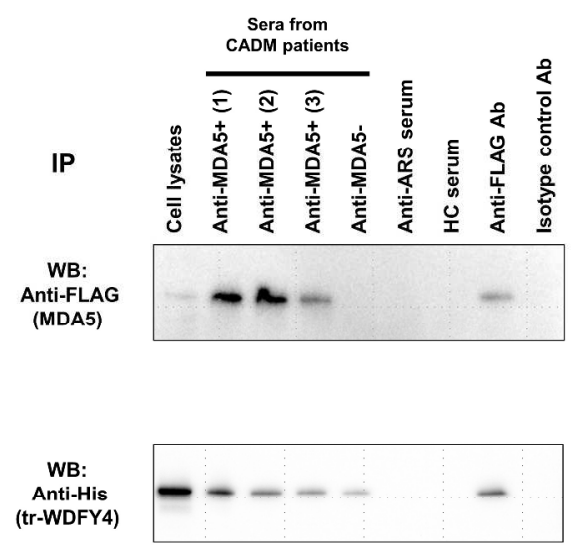

Fig. 5

Fig. 5

$254 \times 381 \mathrm{~mm}(300 \times 300$ DPI $)$

https://mc.manuscriptcentral.com/ard 
Supplementary Table S1 TFs associated with the trans-eQTL effects of WDFY4 variant

\begin{tabular}{|c|c|c|c|c|c|}
\hline TF name & Gene name & $\begin{array}{c}\text { Number of } \\
\text { ChIP-seq data }\end{array}$ & t score & $P$ value & $Q$ value (FDR) \\
\hline HSF1 & HSF1 & 58 & 6.24 & $2.2 \mathrm{E}-10$ & 9.7E-08 \\
\hline HJURP & HJURP & 2 & -13.42 & 2.7E-09 & 5.9E-07 \\
\hline TF65 & RELA & 66 & 5.16 & $1.2 \mathrm{E}-07$ & $1.8 \mathrm{E}-05$ \\
\hline RFX5 & RFX5 & 7 & 3.55 & 0.00019 & 0.021 \\
\hline C2TA & CIITA & 5 & 3.28 & 0.00053 & 0.042 \\
\hline VDR & VDR & 16 & 3.25 & 0.00058 & 0.042 \\
\hline
\end{tabular}

UniProt entry name was used for TFs, and HUGO nomenclature was used for genes. 
Supplementary Table S2 Cis-eQTL effect of rs11101462 for WDFY4

\begin{tabular}{cccccccccc}
\hline SNP & Chr & Pos & $\begin{array}{c}\text { Probe } \\
\text { name }\end{array}$ & $\begin{array}{c}\text { Probe } \\
\text { position }\end{array}$ & $\begin{array}{c}\text { SNP } \\
\text { allele }\end{array}$ & $\begin{array}{c}\text { Allele } \\
\text { assessed }\end{array}$ & Z Score & P value & FDR \\
\hline s1110146; & 10 & $5 \mathrm{E}+07$ & 6620022 & $5 \mathrm{E}+07$ & $\mathrm{~A} / \mathrm{G}$ & $\mathrm{G}$ & 5.34 & $9.24 \mathrm{E}-08$ & $1.61 \mathrm{E}-05$ \\
\hline
\end{tabular}

The data were extracted using the Blood eQTL browser (http://genenetwork.nl/bloodeqtlbrowser/).

The positions of SNP and probe were in the hg19 reference genome. 
All IIM
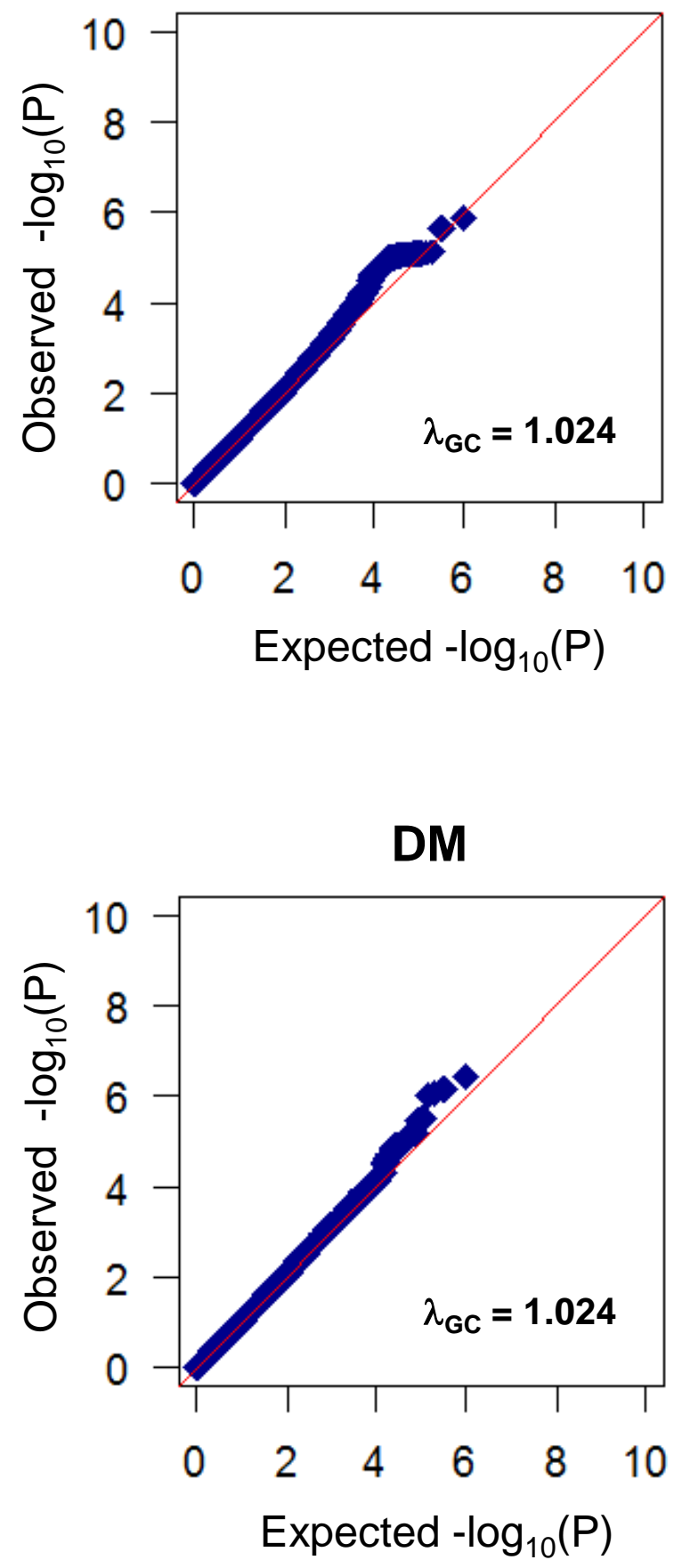

PM

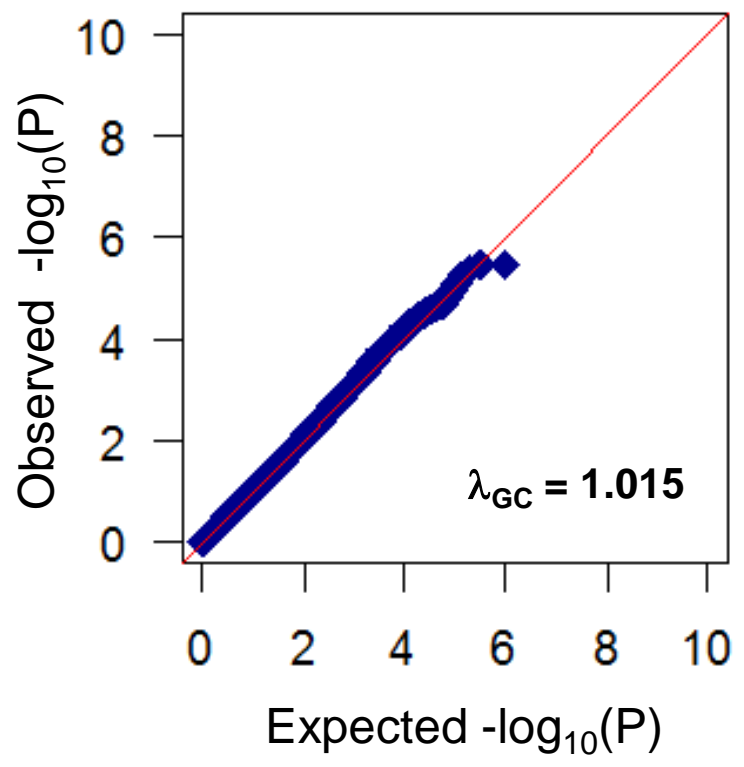

CADM

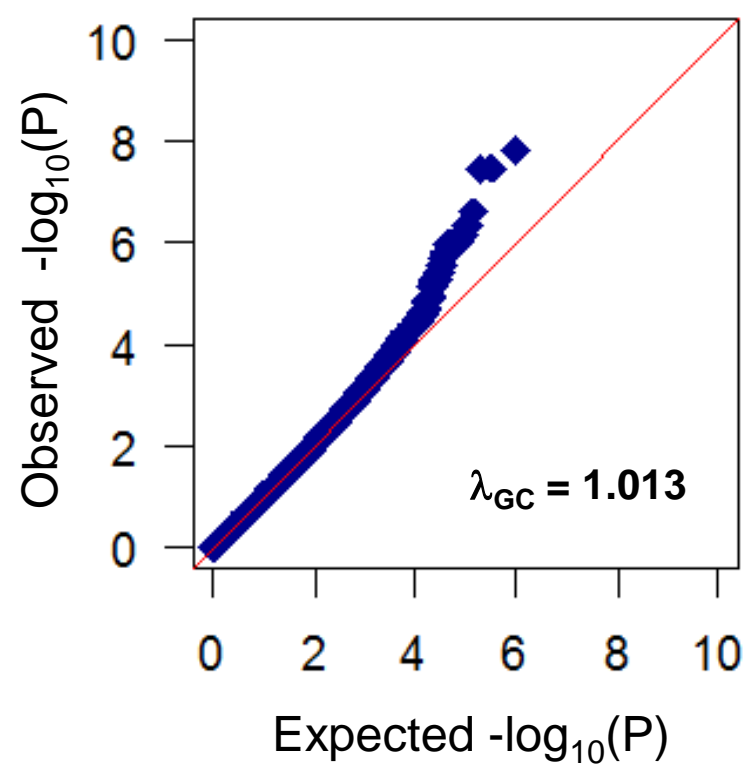

Supplementary Figure S1 Q-Q plot of GWAS for IIM in Japanese.

All IIM, all idiopathic inflammatory myopathies; PM, polymyositis; DM, dermatomyositis; CADM, clinically amyopathic dermatomyositis The observed (y axis) and the expected (x axis) $P$ value is plotted for each SNP (dot), and the red line indicates the null hypothesis of no true association. Genomic inflation factor $\left(\lambda_{G C}\right)$ was indicated. Deviation from the expected $P$-value distribution is evident only in the tail area in CADM, suggesting that population stratification was adequately controlled. 

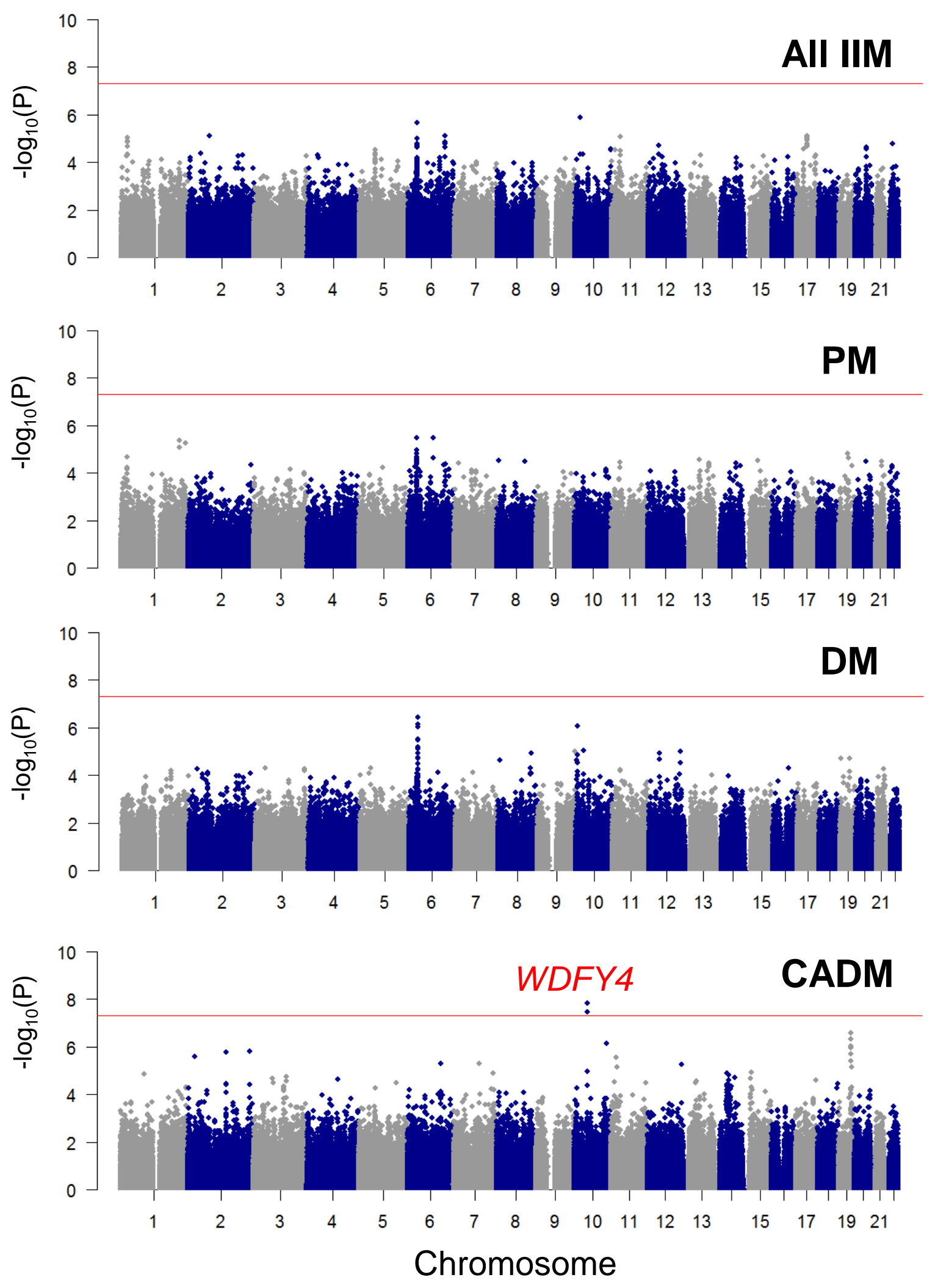

Supplementary Figure S2 Manhattan plots of GWAS for IIMs in Japanese.

All IIM, all idiopathic inflammatory myopathies; PM, polymyositis; DM, dermatomyositis;

CADM, clinically amyopathic dermatomyositis

The image was created using the $R$ package qqman. The red line indicates the genome-wide significance level $\left(P=5 \times 10^{-8}\right)$. Three SNPs (rs7919656, rs3747872 and rs3747874) satisfied the germondewider significamedevedin CADM and were in strong LD with each other $\left(r^{2}>0.95\right)$. 
Supplementary Figure S3. Association of WDFY4 locus with CADM in Europeans. Regional association plot was drawn by LocusZoom. The recombination rate estimate is based on European genotype data from the 1000 Genomes project (hg19 coordinates). The GWAS SNP for CADM in the Japanese (rs7919656) was colored in purple. The LD index $\left(r^{2}\right)$ for each SNP with rs7919656 was also colored as presented in the left panel. The dot line indicates the significance level of $P=5 \times 10^{-8}$ and $P=0.05$. 


\begin{tabular}{|c|c|c|c|c|c|c|c|c|c|c|c|c|c|c|c|c|c|}
\hline pos (hg19) & $\begin{array}{l}\text { LD } \\
\left(r^{2}\right)\end{array}$ & $\begin{array}{l}\text { LD } \\
\text { (D') }\end{array}$ & variant & Ref & Alt & $\begin{array}{l}\text { AFR } \\
\text { freq }\end{array}$ & $\begin{array}{l}\text { AMR } \\
\text { freq }\end{array}$ & $\begin{array}{l}\text { ASN } \\
\text { freq }\end{array}$ & $\begin{array}{l}\text { EUR } \\
\text { freq }\end{array}$ & $\begin{array}{l}\text { SiPhy Promoter } \\
\text { cons histone marks }\end{array}$ & $\begin{array}{l}\text { Enhancer } \\
\text { histone marks }\end{array}$ & DNAse & $\begin{array}{l}\text { Proteins } \\
\text { bound }\end{array}$ & $\begin{array}{l}\text { eQTL } \\
\text { tissues }\end{array}$ & $\begin{array}{l}\text { Motifs } \\
\text { changed }\end{array}$ & $\begin{array}{l}\text { RefSeq } \\
\text { genes }\end{array}$ & $\begin{array}{l}\text { dbSNP } \\
\text { func annot }\end{array}$ \\
\hline chr10:50014917 & 0.85 & 0.96 & $\underline{\text { rs2448540 }}$ & T & c & 0.59 & 0.44 & 0.81 & 0.37 & & 6 organs & & & & 5 altered motifs & WDFY4 & intronic \\
\hline chr10:50015097 & 0.85 & 0.96 & rs2457214 & T & c & 0.65 & 0.46 & 0.81 & 0.37 & & 5 organs & & & & & WDFY4 & intronic \\
\hline chr10:50015211 & 0.84 & 0.95 & rs2448539 & $\mathrm{T}$ & G & 0.64 & 0.54 & 0.81 & 0.51 & & 5 organs & MUS & & & FXR & WDFY4 & intronic \\
\hline chr10:50019099 & 0.9 & 0.98 & $\underline{\text { rs11101511 }}$ & G & c & 0.78 & 0.49 & 0.81 & 0.39 & & ESDR, SKIN, MUS & & & & AP-1 & WDFY4 & intronic \\
\hline chr10:50020323 & 0.89 & 0.98 & $\underline{\text { rs2250389 }}$ & T & c & 0.92 & 0.59 & 0.81 & 0.53 & & SKIN, MUS & & & & HNF4,RXRA & WDFY4 & intronic \\
\hline chr10:50024030 & 0.9 & 0.98 & rs2620896 & c & T & 0.89 & 0.50 & 0.81 & 0.39 & & & & & & Cdc5 & WDFY4 & intronic \\
\hline chr10:50026020 & 1 & 1 & $\underline{\text { rs } 7919656}$ & A & G & 0.57 & 0.55 & 0.82 & 0.51 & & & & & & XBP-1 & WDFY4 & intronic \\
\hline chr10:50029705 & 0.97 & 1 & rs2620891 & A & G & 0.91 & 0.59 & 0.82 & 0.53 & & 4 organs & BRST,SKIN & & & & WDFY4 & intronic \\
\hline chr10:50030864 & 0.97 & 1 & rs 10776647 & c & T & 0.54 & 0.54 & 0.82 & 0.51 & & & & & & EBF,EWSR1-FLI1,TATA & WDFY4 & intronic \\
\hline chr10:50030932 & 0.97 & 1 & rs 10776648 & A & G & 0.57 & 0.55 & 0.82 & 0.51 & & & BLD & & & Foxa,Nanog,SP1 & WDFY4 & intronic \\
\hline chr10:50037720 & 0.97 & 1 & $\underline{\mathrm{rs} 2663051}$ & G & c & 0.91 & 0.59 & 0.82 & 0.53 & & & & & & Mrg,SZF1-1,Spz1 & WDFY4 & intronic \\
\hline chr10:50037974 & 0.97 & 1 & $\underline{\mathrm{rs} 11101529}$ & G & A & 0.71 & 0.56 & 0.82 & 0.51 & & & & & & TCF4 & WDFY4 & intronic \\
\hline chr10:50038285 & 0.95 & 1 & $\underline{\text { rs } 11101530}$ & G & A & 0.50 & 0.43 & 0.81 & 0.36 & & & & & & AP-1,Pax-5 & WDFY4 & intronic \\
\hline chr10:50038487 & 0.95 & 1 & $\underline{\text { rs } 10857655}$ & A & $\mathrm{T}$ & 0.50 & 0.43 & 0.81 & 0.36 & & & & & & AP-1,HNF4 & WDFY4 & intronic \\
\hline chr10:50038585 & 0.95 & 1 & rs3747877 & G & A & 0.54 & 0.44 & 0.81 & 0.36 & & & & & & $\mathrm{CDP}, \mathrm{GR}$ & WDFY4 & intronic \\
\hline chr10:50038720 & 0.95 & 1 & rs 3747875 & G & T & 0.36 & 0.41 & 0.81 & 0.36 & & & & & & Rad21,YY1 & WDFY4 & intronic \\
\hline chr10:50038800 & 0.97 & 1 & rs3747874 & A & G & 0.71 & 0.56 & 0.82 & 0.51 & & & & & & 4 altered motifs & WDFY4 & synonymous \\
\hline chr10:50039253 & 0.95 & 1 & rs7079766 & G & T & 0.50 & 0.43 & 0.81 & 0.36 & & & & & & & WDFY4 & intronic \\
\hline chr10:50039528 & 0.95 & 1 & rs7093474 & c & T & 0.50 & 0.43 & 0.81 & 0.36 & & & & & & Pou5f1 & WDFY4 & intronic \\
\hline chr10:50040076 & 0.8 & 1 & rs7908454 & G & A & 0.70 & 0.52 & 0.79 & 0.50 & & & & & & & WDFY4 & intronic \\
\hline chr10:50040562 & 0.95 & 1 & $\underline{\mathrm{rs} 3747873}$ & A & G & 0.50 & 0.43 & 0.81 & 0.36 & & & & & & 6 altered motifs & WDFY4 & intronic \\
\hline chr10:50040610 & 0.95 & 1 & $\underline{\text { rs3747872 }}$ & T & G & 0.50 & 0.43 & 0.81 & 0.36 & & & BLD & & & 9 altered motifs & WDFY4 & intronic \\
\hline chr10:50041123 & 0.95 & 1 & rs7900138 & A & G & 0.50 & 0.43 & 0.81 & 0.36 & & & & & & GATA,Mef2,SIX5 & WDFY4 & intronic \\
\hline chr10:50041176 & 0.92 & 1 & rs7913005 & G & A & 0.49 & 0.43 & 0.81 & 0.36 & & & & & & 5 altered motifs & WDFY4 & intronic \\
\hline chr10:50041276 & 0.97 & 1 & rs2620898 & G & A & 0.91 & 0.59 & 0.82 & 0.53 & & & & & & 6 altered motifs & WDFY4 & intronic \\
\hline chr10:50041463 & 0.95 & 1 & $\underline{\mathrm{rs} 11101531}$ & T & c & 0.50 & 0.43 & 0.81 & 0.36 & & & & & & BCL,Brachyury,YY1 & WDFY4 & intronic \\
\hline chr10:50041729 & 0.95 & 1 & rs 11101532 & c & A & 0.50 & 0.42 & 0.81 & 0.36 & & & & & & Pitx2,Sox & WDFY4 & intronic \\
\hline chr10:50041734 & 0.97 & 1 & $\underline{\text { rs22448545 }}$ & c & G & 0.94 & 0.59 & 0.82 & 0.53 & & & & & & EBF,Pitx2,TCF12 & WDFY4 & intronic \\
\hline chr10:50042319 & 0.95 & 1 & $\underline{\text { rs } 58214108}$ & A & G & 0.50 & 0.43 & 0.81 & 0.36 & & MUS & & & & 7 altered motifs & WDFY4 & intronic \\
\hline chr10:50042579 & 0.95 & 1 & rs 3860188 & T & c & 0.50 & 0.43 & 0.81 & 0.36 & & MUS & & & & Esr2,Hdx & WDFY4 & intronic \\
\hline chr10:50042951 & 0.95 & 1 & $\underline{\text { rs877819 }}$ & A & G & 0.54 & 0.44 & 0.81 & 0.36 & & PANC & & & & 4 altered motifs & WDFY4 & intronic \\
\hline chr10:50043141 & 0.95 & 1 & rs877820 & A & G & 0.50 & 0.43 & 0.81 & 0.36 & & PANC & & & & 4 altered motifs & WDFY4 & intronic \\
\hline chr10:50043317 & 0.94 & 1 & $\underline{\text { rs877821 }}$ & G & c & 0.17 & 0.39 & 0.81 & 0.36 & & PANC & & & & 18 altered motifs & WDFY4 & intronic \\
\hline chr10:50043766 & 0.94 & 1 & rs3860189 & A & G & 0.50 & 0.43 & 0.81 & 0.36 & & ESDR & & & & 4 altered motifs & WDFY4 & intronic \\
\hline chr10:50043857 & 0.95 & 1 & $\underline{\text { rs } 3860190}$ & T & c & 0.50 & 0.43 & 0.81 & 0.36 & & ESDR & & & & Evi-1,Foxp1,SIX5 & WDFY4 & intronic \\
\hline chr10:50043889 & 0.95 & 1 & rs2002588 & A & G & 0.50 & 0.43 & 0.81 & 0.36 & & ESDR & BRN & & & Pousf1 & WDFY4 & intronic \\
\hline chr10:50043907 & 0.95 & 1 & rs2002587 & T & c & 0.50 & 0.43 & 0.81 & 0.36 & & ESDR & BRN,BRN & & & 8 altered motifs & WDFY4 & intronic \\
\hline chr10:50044824 & 0.95 & 1 & rs6537580 & G & A & 0.50 & 0.43 & 0.81 & 0.36 & & MUS & & & & GATA, Gfi1, Hoxa7 & WDFY4 & intronic \\
\hline chr10:50045021 & 0.97 & 1 & rs2620904 & G & C & 0.91 & 0.59 & 0.82 & 0.53 & & MUS & & & & Foxl1,Foxo & WDFY4 & intronic \\
\hline chr10:50072102 & 0.91 & 0.96 & rs2940727 & G & A & 0.91 & 0.59 & 0.82 & 0.53 & & & BLD & & & 4 altered motifs & WDFY4 & intronic \\
\hline chr10:50080311 & 0.88 & 0.98 & rs1904596 & A & G & 0.96 & 0.63 & 0.81 & 0.59 & & & & & & 8 altered motifs & WDFY4 & intronic \\
\hline
\end{tabular}

Supplementary Figure S4 Candidate causal variants searched from the 1000 genome data by using HaploReg.

All the variants in LD $\left(r^{2}>=0.8\right.$ in Asian samples) with the GWAS SNP (rs7919656) were shown. No missense variant was identified. HaploReg, http://archive.broadinstitute.org/mammals/haploreg/haploreg.php. 


\section{Exon 35}

$$
\begin{aligned}
& \beta=-2.8 \\
& P=0.18
\end{aligned}
$$

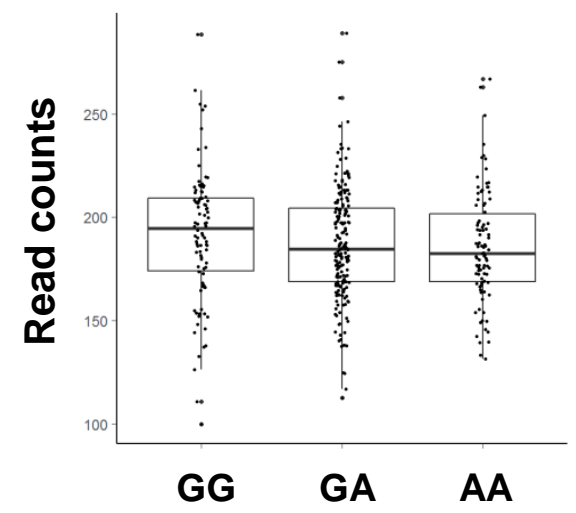

Exon 37

$$
\begin{aligned}
& \beta=-0.87 \\
& P=0.62
\end{aligned}
$$

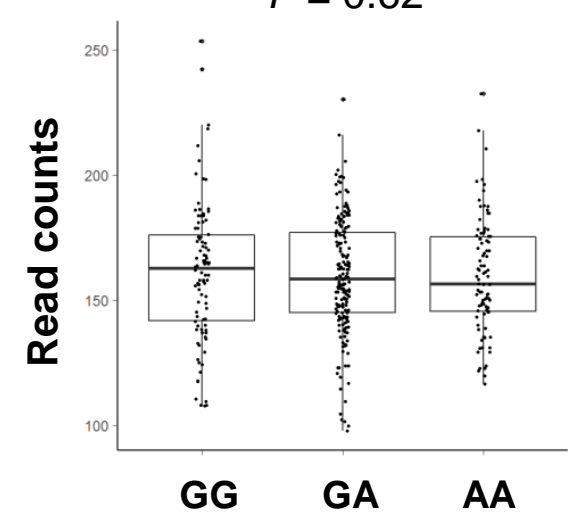

\section{Exon 36}

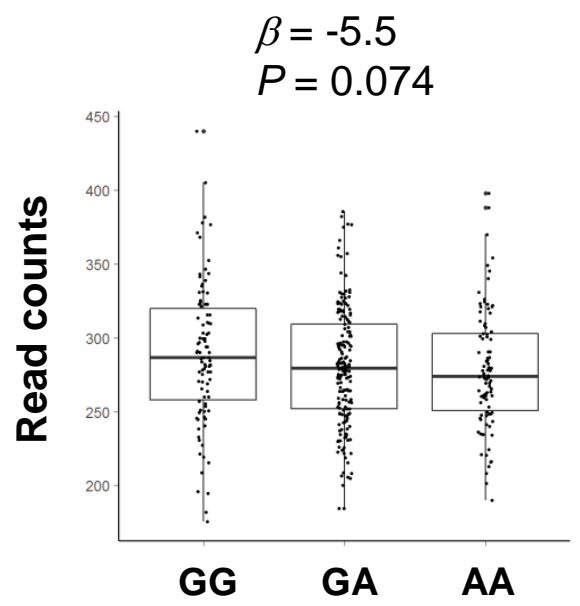

New exon

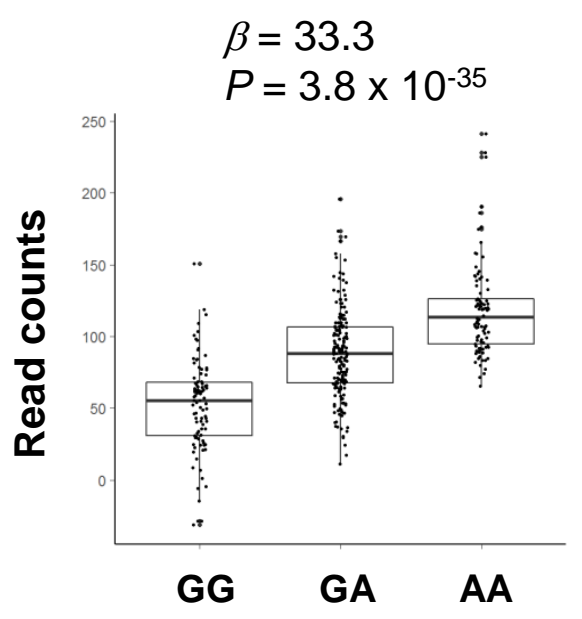

Supplementary Figure S5 Cis-eQTL analysis of WDFY4 variant (rs7919656) with the expression levels of exons using the Guevadis project samples $(N=373)$. The line in the middle of the box is the median, and the box edges are the 25th and 75th percentiles. 


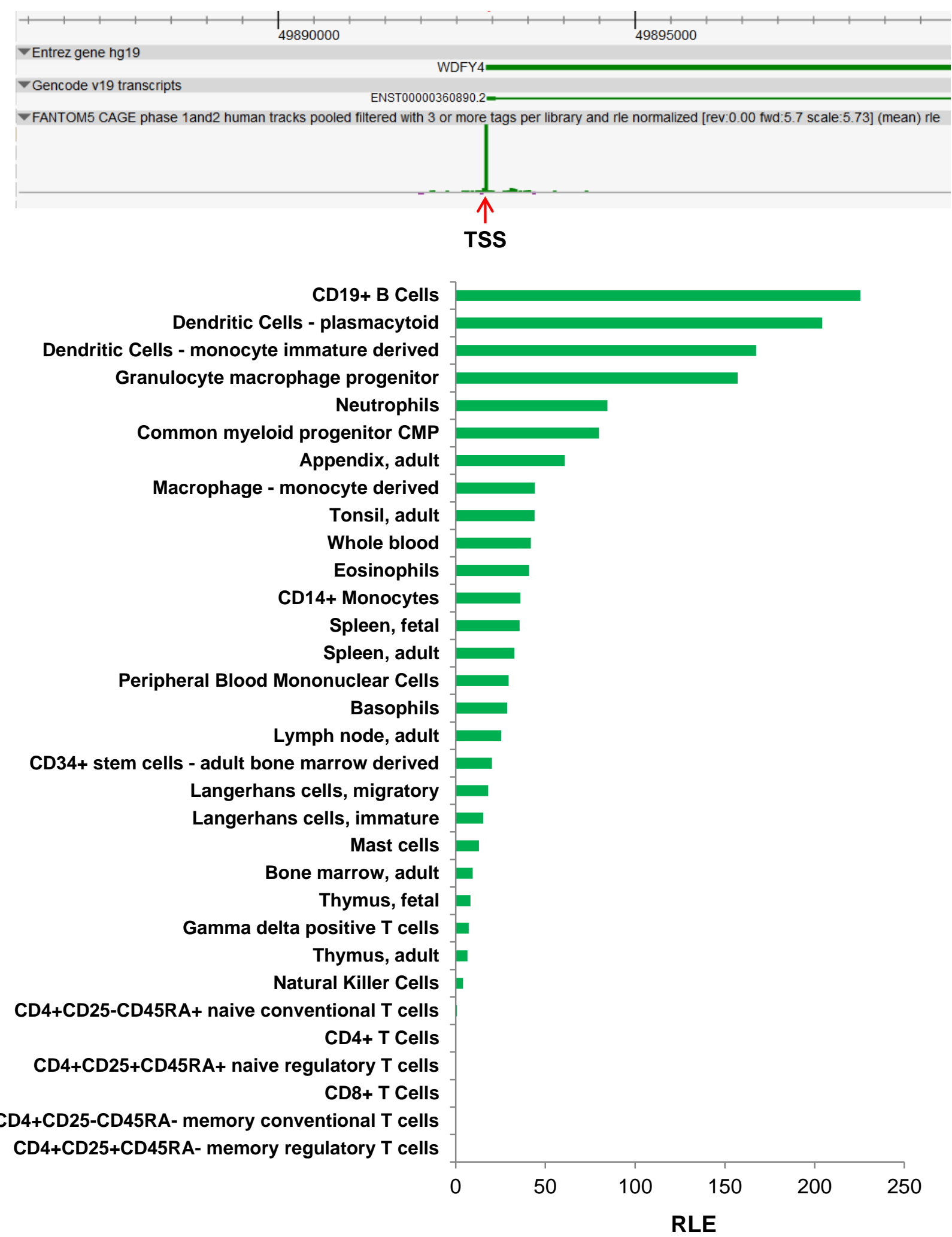

Supplementary Figure S6 Expression levels of WDFY4 in immune cells and tissues. The expression data of WDFY4 were extracted from the FANTOM5 database using ZENBU. The transcriptional start site (TSS) expression was quantified by cap analysis of gene expression (CAGE) and displayed as relative log expression (RLE). ZENBU, http://fantom.gsc.riken.jp/zenbu/. 
Intervention
(stimulation etc)

\section{State 2}

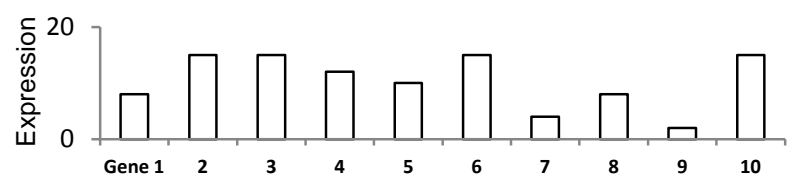

State 1 / state2 expression ratio

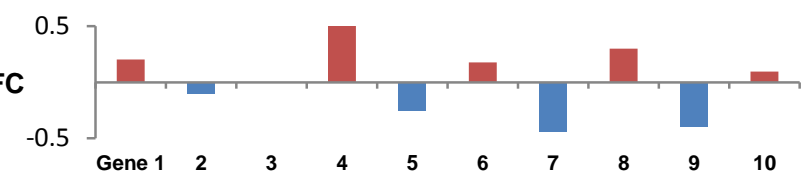

Log fold change

(LFC)
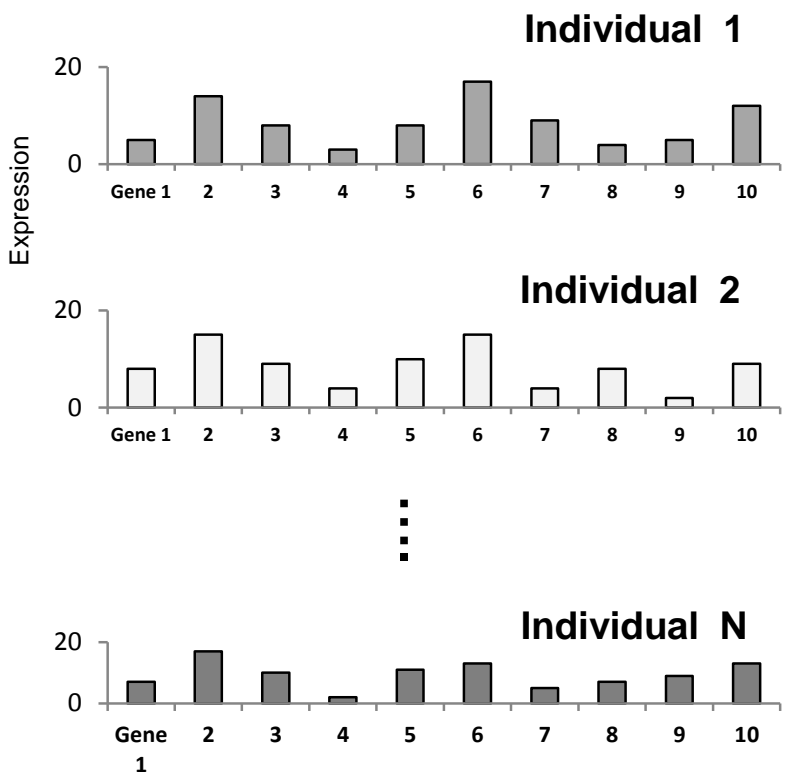

$\mathrm{Y}=\alpha+\beta \mathrm{X}$

$Y$ : expression

$X:$ SNP genotype

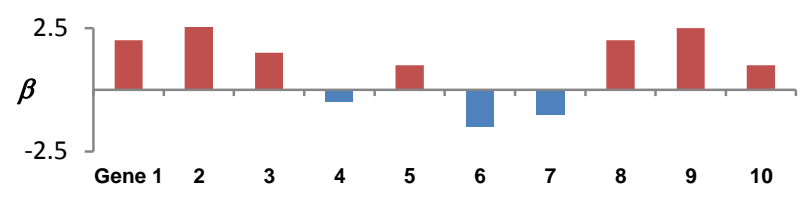

Trans-eQTL effect

$(\beta)$
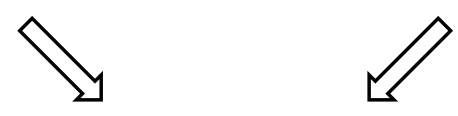

TF

ChIP-seq

data

Gene set enrichment analysis

wPGSA

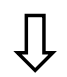

Responsible TFs for the regulation

Supplementary Figure $\mathbf{S 7}$ Application of the results of trans-eQTL analysis to wPGSA. Log-fold change (LFC) of expression data is usually evaluated in wPGSA. Instead, we evaluated trans-eQTL effects $(\beta)$ of a variant for distal genes and identified the transcriptional factors responsible for the regulation. 


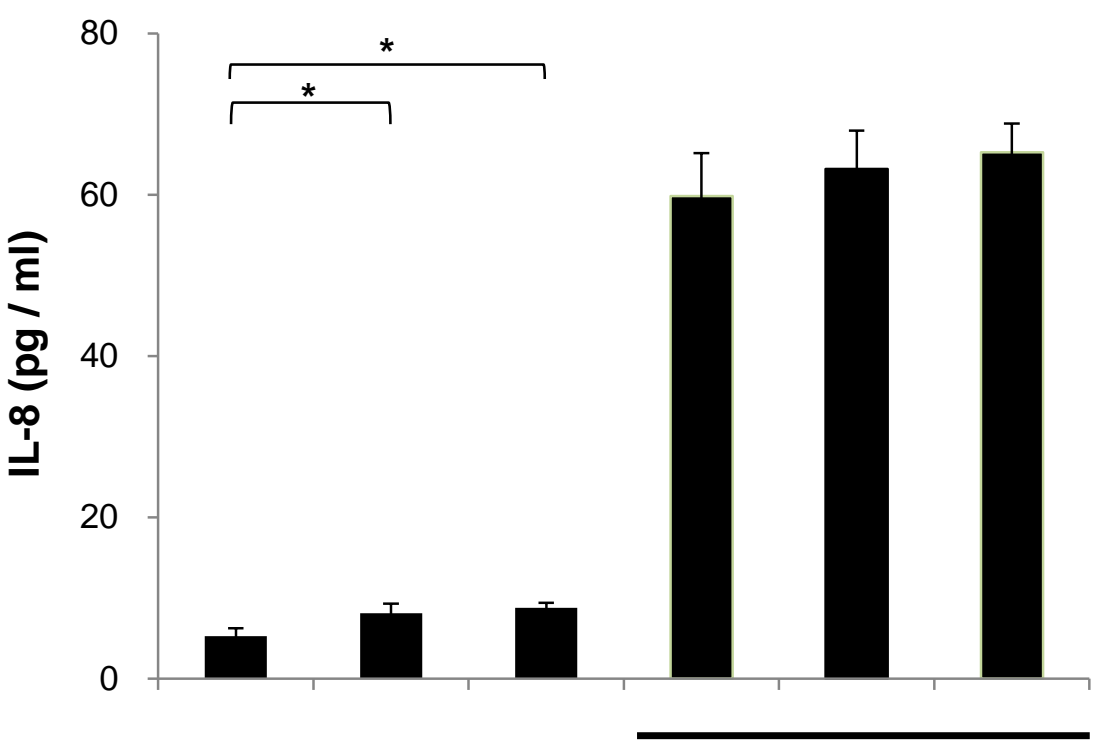

HMW Poly (I:C)

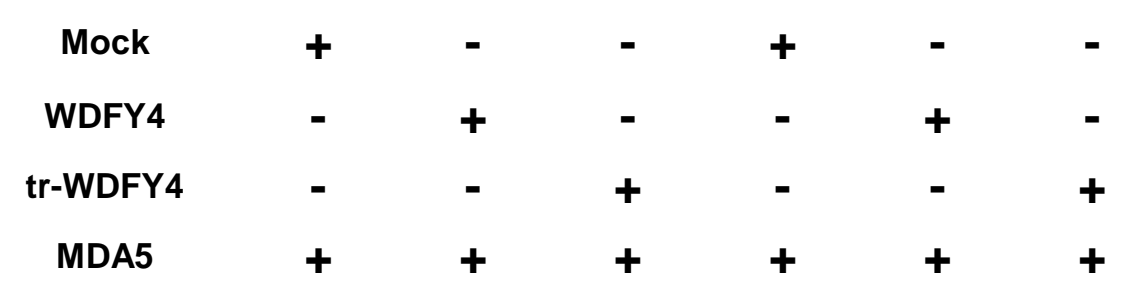

Supplementary Figure S8 Impact of WDFY4 isoforms on cytokine production in HEK-293 cells. HEK-293 cells were transfected with WDFY4 isoforms and MDA5 with or without stimulation by high molecular weight (HMW) poly (I:C) at $1 \mu \mathrm{g} / \mathrm{ml}$ for 24 hours. IL-8 in the cell culture supernatant was quantified by human IL-8 ELISA MAX kit (BioLegend) in a sandwich ELISA. Means and s.d. are shown for 6 technical replicates. ${ }^{*} P<0.05$ by ANOVA with post-hoc Tukey's test. 